\title{
Searching for a new probability distribution for modeling non- scale-free heavy-tailed real-world networks
}

This paper was downloaded from TechRxiv (https://www.techrxiv.org).

\section{LICENSE}

CC BY 4.0

SUBMISSION DATE / POSTED DATE

23-01-2022 / 29-01-2022

\section{CITATION}

CHAKRABORTY, TANUJIT; Chattopadhyay, Swarup; Das, Suchismita; Kumar, Uttam; Jayavelu, Senthilnath (2022): Searching for a new probability distribution for modeling non-scale-free heavy-tailed real-world networks. TechRxiv. Preprint. https://doi.org/10.36227/techrxiv.18934175.v1

$\mathrm{DOI}$ 


\title{
Searching for a new probability distribution for modeling non-scale-free heavy-tailed real-world networks
}

\author{
Tanujit Chakraborty ${ }^{1, *}$, Swarup Chattopadhyay ${ }^{2}$, Suchismita Das ${ }^{3}$, Uttam Kumar ${ }^{1}$, J. \\ Senthilnath $^{4}$ \\ 1 Spatial Computing Laboratory, Center for Data Sciences, International Institute of \\ Information Technology Bangalore, India - 560100 \\ 2 Machine Intelligence Unit, Indian Statistical Institute, Kolkata, India - 700108 \\ 3 Department of Data Science, S P Jain School of Global Management, Mumbai, India - \\ 400070 \\ 4 Institute for Infocomm Research, Agency for Science, Technology and Research, \\ Singapore - 138632 \\ * tanujit.c@iiitb.ac.in
}

\begin{abstract}
Perhaps the most controversial topic in network science research is whether real-world complex networks are scale-free or not. Recently, Broido and Clauset [A.D. Broido, A. Clauset, Nature Communication, 10, 1017 (2019)] claimed that the degree distributions of real-world networks are rarely power-law under statistical tests. Such complex networks include social, biological, information, temporal, and brain networks are often heavy-tailed where the assumption on scale-free nature of real-world heavy-tailed networks becomes insignificant so as the complex system evolves over time. The failure of power-law distribution in fitting the degree distribution data is mainly due to the presence of an identifiable non-linearity in the entire degree distribution in a log-log scale of a heavy-tailed complex network. Here, we attempt to address this issue by proposing a new class of heavy-tailed probability distributions, for modeling the entire degree distributions of complex networks and capturing the non-linearity of these heavy-tailed networks. The generalized Lomax Model is introduced to fit the degree distributions of non-scale-free real-world networks and applied to model a wide variety of large-scale real-world complex networks. Several statistical properties of the proposed model, such as extreme value and inferential statistical properties are derived into this context. Rigorous experimental analysis showcases the excellent performance of the proposed family of distributions while fitting the heavy-tailed real-world complex networks over fifty real-world data sets in comparison with the state-of-the-art.
\end{abstract}

\section{Introduction}

The structure of a complex system can be well studied by representing the system as a network. As it is known, most of the practical networks such as communication, traffic, spatial, social, temporal, and biological networks are regarded as complex systems with the same properties as heavy-tailed and large-scale [1 3]. Recent research across the field of networks focuses on justifying the common claim about the scale-free property of real-world complex networks 4 [6]. We call a network distribution scale-free when its degree distribution follows power-law. One of the most important structural 


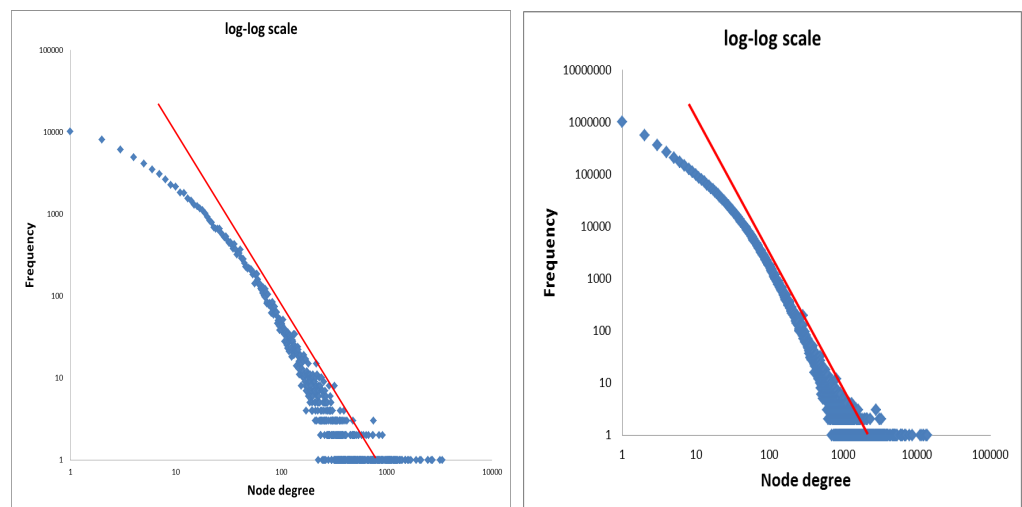

Fig 1. Plots of degree distributions of Twitter and Livejournal network data to show the non-scale-free nature of these heavy-tailed networks.

characteristics of large-scale real-world networks is degree distribution which characterize a network system $7 \cdot 9]$. Empirical studies in the last two decades on real-world complex networks such collaboration, communication, social, biological and temporal networks are assumed to follow a power-law distribution 8,1013 .

Mathematically, a random variable $X$ is said to follow the power-law model if its probability distribution is of the form $P(x) \propto x^{-\alpha}$, where $\alpha$ is a positive constant and is known as the tail-index or shape parameter of the distribution. Therefore, it is common to claim the scale-free property of real-world networks that the degree distributions of such networks follow a single power-law.

In Figure 1, a closer look at the plots of entire degree distributions for Twitter and Livejournal networks (in log-log scale) offers clear evidence of the presence of an identifiable non-linearity (bend) into it. This fact suggests that a single power-law is inappropriate to fit the entire degree distribution data. Consequently, the current researchers also reported that a baseline power-law is insufficient to properly fit the empirical data in its whole range unless some of the lower degree nodes are left out while model fitting 11, 14 17]. Recently, Broido \& Clauset (2019) [18] mentioned that (strict) scale-free networks are rare, which relate to the claims of Stumpf et al. (2005) 19, that all the derived sub-networks of a scale-free network fails to meet the scale-free property. Apart from power-law, researchers have also proposed various heavy-tailed distributions such as lognormal, Pareto lognormal, and double Pareto lognormal for modeling the degree distribution of real-world complex networks [20,21. Despite the alternatives, the long-tailed and heavy-tailed behavior of the entire degree distribution of complex real-world networks remains a challenging task in network science, even as it slowly moves toward the fields of modern statistics and data science [15,22, 23 .

\section{Motivation}

Over the last two decades, a remarkable amount of research focuses on studying heavy-tailed complex networks in physical, biological, social, and network sciences. A scale-free network is a network whose degree distribution follows a power-law, at least asymptotically. While studying internet networks, Barabási \& Albert (1999) [9] observed that some nodes, called hubs, had many more connections than others and coined the term scale-free network to describe that class of networks having a power-law degree distribution. Clauset, Cosma \& Newman (2007) 11 studied real networks and found that some of the complex networks exhibit the same property. Nevertheless, recently, Broido \& Clauset (2019) [18] claimed that (strict) scale-free network are 
actually rare. More specifically, Kaviani et al. (2021) [24 surveyed various notions of weak or strong scale-free networks. Voitalov et al. (2018) 15 showed that the scale-free property is closely related to the Pareto type-II or Lomax distribution. Broido \& Clauset (2019) 18, also observed that the recent data concentration on complex network data sets shows that they no longer follow the power-law distribution (scale-free nature), which relates to the earlier claim of Stumpf, Wiuf \& May (2005) 19].

The log-log scale plot of the degree distribution of the Livejournal and Twitter networks in Figure 1 shows the unique degree values $(x)$ presented in the horizontal $x$ axis and the corresponding frequency presented in the vertical $y$ axis. A node corresponding to these networks represent a single user and follower of that user is represented by an edge in the network. Figure 1 shows that the straight-line representation using a single power-law fails to fit the degree distribution data in the log-log scale. Generally, a single power-law distribution is applied to fit the degree distribution data only when the values of degree are considered higher than $x_{\text {min }}$ (minimum degree). Subsequently, the power-law exponent $\alpha$ is estimated using maximum likelihood estimation (MLE) from the data with the help of $x_{\text {min }}$. This intern suggests that the power-law distribution tends to provide a better fit only when some lower-degree nodes are left out. The insufficiency of such fitting using a single power-law is due to the presence of a non-linearity in the log-log scale of the degree distribution of a complex network 18, 19] as depicted in Figure 1. Such a drawback inspires many researchers to use other heavy-tailed probability models with various exponents for better fitting the entire degree distribution of real-world complex networks. This article proposes a new class of generalized Lomax models for modeling these heavy-tailed real-world complex networks. The non-negative shape parameter of these new family of distributions can be expressed as a nonlinear function of the network data sets.

\section{Contribution}

Motivated by the above discussion, this article introduces a "generalized Lomax models" (GLM), a new class of heavy-tailed probability distributions, to describe real networks than the strict scale-free property. In generalized Lomax models, the non-negative shape parameter (tail-index) is supposed to be defined as a nonlinear function of the degree distribution data. An apparent reason for introducing the GLM family compared to other popularly used heavy-tailed distributions is for better fitting and gaining greater flexibility to the entire node degree distribution of complex networks.

Alternatively, the proposed generalized Lomax model can be effectively used to model the whole degree distribution without discarding any lower degree nodes of a complex network coming from different disciplines.

The proposed GLM family is closely related to various life distributions such as Exponential, Weibull, and Rayleigh distributions. We study some specific models and few extreme value properties of this new family of distributions. We have theoretically shown that the models of the GLM family belong to the basin of attractions of the Frechet family of distributions, possess heavy-tails and long-tails, and tail-equivalent to Pareto distribution. We also discuss parameter estimation procedures and goodness of fit for the newly introduced GLM family of distributions. Some nonlinear variants of the proposed GLM family provide better fits than the other popular heavy-tailed models studied over fifty real-world complex network data sets. The new family of proposed GLM distributions is compared with several other popularly used distributions, such as Pareto, Exponential, log-normal, and power-law with an exponential cutoff model 25,26]. The standard statistical measures, viz. Kullback-Leibler divergence (KLD), mean absolute error (MAE), and root mean square error (RMSE) has been used to evaluate the goodness-of-fit test. Empirical results confirm the effectiveness of the new GLM family of distributions compared to other 
similar types of distributions in terms of the goodness-of-fit from an application point of view. This newly introduced 'shape-parameter-based generalization approach' for the Lomax distribution can be seen as one such class of distributions that can better fit the entire degree distributions of heavy-tailed networks in general.

The organization of the rest of the paper is as follows. The basic notion of the Lomax distributions is discussed in Section . We have introduced the new family of GLM distributions in Section. Section discusses some statistical properties of the new class of the GLM family. Experimental analysis of over fifty real-world complex networks is provided in Section. Finally Section concludes the paper.

\section{Preliminaries}

K.S. Lomax in 1954 first proposed the Lomax distribution for modeling business failures 27. The Lomax distribution is one of the popularly and widely used heavy-tailed distribution, which has applications in the field of economics, actuarial science, life testing, reliability modeling, operation research, and network analysis 27 30. Lomax distribution closely resembles a Pareto type II distribution with support beginning at zero 27], and it can be motivated in several ways from the theoretical and application point of view. For example, the Lomax distribution can be derived as a special case of a particular compound gamma distribution [31]. Again the Lomax distribution is also represented as limiting distribution of the residual lifetime at a great age. The record values of the Lomax distribution, as well as the amalgamation of the Lomax distribution with Poisson distribution, were well studied in 32, 33.

Researchers have also studied the possible extensions and various modifications of the Lomax distribution to model the real-life problems 34. Recent studies 35 suggest that the Lomax distribution is used for the analysis of heavy-tailed data and can make use of an alternative to the gamma, Exponential, and Weibull distributions. The corresponding probability density function (PDF) and cumulative distribution function (CDF) of the Lomax model are defined as follows:

Definition 1. A random variable $X$ follows Lomax distribution with parameters $\alpha, \gamma$, which is denoted by $\operatorname{LM}(\alpha, \gamma)$ if the CDF is of the following form:

$$
F(x ; \alpha, \gamma)=1-\left(1+\frac{x}{\gamma}\right)^{-\alpha} ; x>0,
$$

where $\alpha(>0)$ is a shape parameter (real) and $\gamma(>0)$ is a scale parameter (real).

The PDF for the Lomax distribution is given by

$$
f(x ; \alpha, \gamma)=\frac{\alpha}{\gamma}\left(1+\frac{x}{\gamma}\right)^{-\alpha-1} ; x>0
$$

with mean $=\frac{\gamma}{\alpha-1}, \alpha>1$ and variance $=\frac{\alpha \gamma^{2}}{(\alpha-1)^{2}(\alpha-2)}, \alpha>2$.

The quantile function is given by

$$
Q(u ; \alpha, \gamma)=\gamma\left[(1-u)^{-1 / \alpha}-1\right]
$$

and the expression for the $r^{t h}$ order moment of the Lomax distribution is as follows 36 :

$$
E\left[X^{r}\right]=\frac{\alpha \gamma^{r} \Gamma(r+1) \Gamma(\alpha-r)}{\Gamma(\alpha+1)} ; \alpha>r, r=1,2,3, \ldots
$$


The survival function is given by

$$
S(x ; \alpha, \gamma)=\left(1+\frac{x}{\gamma}\right)^{-\alpha} ; x>0 .
$$

The hazard function is given by

$$
h(x ; \alpha, \gamma)=\frac{\alpha}{\gamma}\left(1+\frac{x}{\gamma}\right)^{-1} ; x>0
$$

which is a decreasing function of $x$.

It is interesting to see that the Exponential distribution arises as a limit of the Lomax distribution when the shape parameter or tail-index $(\alpha)$ increases. This can be seen through a re-parameterization of

$$
\gamma=\frac{\alpha\left(1+o_{\alpha}(1)\right)}{\lambda},
$$

where $o_{\alpha}(1) \rightarrow 0$ as $\alpha \rightarrow \infty$ and $\lambda>0$. Thus, we have

$$
\begin{aligned}
\lim _{\alpha \rightarrow \infty} P(X>x) & =\lim _{\alpha \rightarrow \infty}\left(1+\frac{x}{\gamma}\right)^{-\alpha} \\
& =\lim _{\alpha \rightarrow \infty}\left(1+\frac{\lambda x}{\alpha\left(1+o_{\alpha}(1)\right)}\right)^{-\alpha} \\
& =e^{-\lambda x} ; \lambda>0 .
\end{aligned}
$$

We can conclude that the limiting distribution of the Lomax distribution for $\alpha \rightarrow \infty$ is ${ }^{124}$ the Exponential distribution [32]. However, the Lomax distribution fails to provide ${ }_{125}$ greater flexibility while modeling heavy-tailed data sets in its whole range. In this ${ }_{126}$ paper, we introduce a new method of generalizing Lomax distributions by modeling the ${ }^{127}$ tail index of the Lomax model for fitting the entire degree distributions of real-world ${ }_{128}$ heavy-tailed networks.

\section{Proposed Model}

An essential structural characteristic in the study of heavy-tailed real-world complex networks is their degree distributions. Empirical observations in the analysis of the pattern of real-world complex networks have led to the claim that their degree distributions follow, in general, a single power-law. However, while fitting, a closer observation suggests that the single power-law distribution is inappropriate to model the network data in its whole range. We first introduce a new class of generalized Lomax models (GLM) whose members belong to a maximum domain of attraction of the Frechet distribution and are right tail equivalent to Pareto distribution. These newly introduced heavy-tailed families will suffice the need for new probability distributions for modeling network data.

\section{Family of Generalized Lomax Models (GLM)}

We consider a real, continuous, and positive function $g:(0, \infty) \rightarrow \mathcal{R}^{+}$which is differentiable on $(0, \infty)$. Also, we assume that $g$ satisfies conditions stated below.

1. $\lim _{z \rightarrow 0^{+}}(1+z)^{g(z)}=1$;

2. $\lim _{z \rightarrow \infty}(1+z)^{g(z)}=\infty$; 
3. $\frac{g^{\prime}(z)}{g(z)} \geq \frac{-1}{(1+z) \log (1+z)}, z>0$,

where $g^{\prime}(z)$ is the first-order derivative of $g(z)$.

It is noted that the condition 3 is equivalent to

$$
\frac{d}{d z} \log (g(z)) \geq-\frac{d}{d z} \log [\log (1+z)], z>0 .
$$

Then, for any function $g$ with a strictly positive and finite limit at infinity

$$
\lim _{z \rightarrow \infty} g(z)=\alpha>0
$$

and satisfying the above three conditions, we define the new class of GLM distributions as follows:

$$
F(x)= \begin{cases}1-\left(1+\frac{x}{\gamma}\right)^{-g(x / \gamma)}, & \text { if } x>0 \\ 0, & \text { if } x \leq 0\end{cases}
$$

where, $\gamma(>0)$ is a scale parameter.

It is very easy to verify that:

1. $F(x)$ is non-decreasing since

$$
g\left(\frac{x}{\gamma}\right)+\left(1+\frac{x}{\gamma}\right) \log \left(1+\frac{x}{\gamma}\right) g^{\prime}\left(\frac{x}{\gamma}\right)>0 \text { for } x>0 .
$$

2. $\lim _{x \rightarrow-\infty} F(x)=F(-\infty)=0$ and $F(\infty)=1$.

3. $F(x)$ is right continuous.

Thus, $F(x)$ is a standard CDF and any continuous random variable $X$ satisfying the above-mentioned conditions are called class of GLM distributions. The CDF of standard GLM distributions can also be expressed as follows:

$$
F(x)=1-\exp \left[-g\left(\frac{x}{\gamma}\right) \log \left(1+\frac{x}{\gamma}\right)\right], x>0 .
$$

The survival function is given by

$$
S(x)= \begin{cases}\left(1+\frac{x}{\gamma}\right)^{-g(x / \gamma)}, & \text { if } \quad x>0 \\ 1, & \text { if } \quad x \leq 0\end{cases}
$$

For GLM family of distributions, the survival distribution function decrease as a power function. The probability density function (pdf) of GLM is

$$
f(x)= \begin{cases}\frac{S(x)}{\gamma}\left[\frac{g(x / \gamma)}{(1+x / \gamma)}+g^{\prime}\left(\frac{x}{\gamma}\right) \log \left(1+\frac{x}{\gamma}\right)\right], & x>0 \\ 0, & x \leq 0 .\end{cases}
$$

The hazard function is given by

$$
h(x)= \begin{cases}\frac{1}{\gamma}\left[\frac{g(x / \gamma)}{(1+x / \gamma)}+g^{\prime}\left(\frac{x}{\gamma}\right) \log \left(1+\frac{x}{\gamma}\right)\right], & \text { if } x>0 \\ 0, & \text { if } x \leq 0 .\end{cases}
$$


Table 1. Some examples of GLM family of distributions (with $\lim _{z \rightarrow \infty} g(z)=\alpha>0$ ).

\begin{tabular}{|c|c|c|c|c|c|c|c|c|}
\hline \multicolumn{2}{|r|}{$g(z), \forall z>0$} & $\beta$ & \multicolumn{2}{|l|}{ Distribution } & \multicolumn{4}{|c|}{$f(x), \forall x>0$} \\
\hline \multicolumn{2}{|r|}{$\alpha$} & & \multicolumn{2}{|l|}{ Lomax } & \multicolumn{4}{|c|}{$\frac{\alpha}{\gamma}\left[1+\frac{x}{\gamma}\right]^{-(\alpha+1)}$} \\
\hline \multirow{2}{*}{$\alpha$} & $\beta$ & \multirow{2}{*}{$\beta \geq-1$} & \multirow{2}{*}{\multicolumn{2}{|c|}{ GLM Type-I }} & \multirow{2}{*}{$\stackrel{\alpha}{-}\left[1+\frac{x}{-}\right]^{-(\alpha+1)}$} & \multirow{2}{*}[1+\frac{\beta}{(1+\operatorname{log}(1+x/\gamma))^{2}}]{$\exp$} & \multirow{2}{*}{\multicolumn{2}{|c|}{$\mathrm{p}\left[-\alpha \beta \frac{\log (1+x / \gamma)}{1+\log (1+x / \gamma)}\right]$}} \\
\hline & $\overline{1+\log (1+z)}]$ & & & & & & & \\
\hline \multirow{2}{*}{$\alpha[$} & $\beta z$ & \multirow{2}{*}{$\beta \geq-1$} & \multirow{2}{*}{\multicolumn{2}{|c|}{ GLM Type-II }} & \multirow{2}{*}{$\frac{\alpha}{\gamma}\left[1+\frac{x}{\gamma}\right]$} & $\beta$ & \multirow{2}{*}{\multicolumn{2}{|c|}{$\left[-\alpha \beta\left(\frac{x / \gamma}{1+x / \gamma}\right)\right]$}} \\
\hline & $+\overline{(1+z) \log (1+z)}]$ & & & & & $\overline{(1+x / \gamma)}]^{\exp }$ & & \\
\hline \multirow{2}{*}{\multicolumn{2}{|c|}{$\alpha\left[\frac{z}{1+z}\right]^{\beta}$}} & \multirow{2}{*}{$\beta>-1$} & \multirow{2}{*}{\multicolumn{2}{|c|}{ GLM Type-III }} & \multirow{2}{*}[1+\frac{\gamma\beta\operatorname{log}(1+x/\gamma)}{x}]{} & {$[x / \gamma]^{\beta}$} & \multirow{2}{*}{\multicolumn{2}{|c|}{$-\alpha \log \left(1+\frac{x}{\gamma}\right)\left(\frac{x / \gamma}{1+x / \gamma}\right)^{\beta}$}} \\
\hline & & & & & & {$[1+x / \gamma]$} & & \\
\hline \multirow{2}{*}{$\alpha[$} & {$[\log (1+z)]^{\beta}$} & \multirow{2}{*}{$\beta>-1$} & \multirow{2}{*}{ GLM Type-IV (MLM } & \multirow{2}{*}{ (17) } & $\alpha[1+x]^{-1}$ & \multirow{2}{*}{$\frac{\log (1+x / \gamma)}{1+\log (1+x / \gamma)}$} & \multirow{2}{*}{$\beta \exp$} & $\log ^{\beta+1}(1+x / \gamma)$ \\
\hline & {$[\overline{1+\log (1+z)}]$} & & & & $(1+\log (1+x / \gamma))$ & & & {$[1+\log (1+x / \gamma)]^{\beta}$} \\
\hline
\end{tabular}

Table 1 shows some examples of GLM family of distributions satisfying limiting condition as in 1 , i.e., $\lim _{z \rightarrow \infty} g(z)=\alpha>0$. It is observable that the Lomax distribution belongs to this family and corresponds to the simplest choice $g(z)=\alpha$. Some of the models are novel among Lomax, GLM Type-I, GLM Type-II, GLM Type-III, and GLM Type-IV distributions presented in Table 1. Out of which GLM Type-IV is very similar to Modified lomax model (MLM) proposed in [16,17]. Though both the models (GLM Type-IV and MLM distributions) are derived from completely different phenomena but achieve the same goal of building a heavy-tailed lomax model for explaining nonlinearity in the degree distributions of the real-world complex network data sets. Other GLM type models are completely new whereas GLM family of distribution covers as particular cases several very popular probability distributions as indicated in Table 2. These models treat the tail index of the Lomax distribution as a nonlinear function of the data with two parameters $\alpha$ and $\beta$. To select a model, one can choose the nonlinear exponent $g(z)$ that meets the empirical characteristics of the given data sets. Some graphics of the new models derived from this newly introduced GLM family of distributions are illustrated in Figures 2, 3, 4, and 5. This example list of GLM models can easily be expanded to more models by introducing some other forms of $g(z)$ satisfying the above-mentioned conditions and $\lim _{z \rightarrow \infty} g(z)=\alpha>0$ or by increasing the number of parameters in the function $g$. The members of this GLM family belong to a maximum domain of attraction of Frechet distribution and are heavy-tailed, as shown in Section .

\section{Closeness of GLM and Life distributions}

We provide some examples of an extended family of GLM distributions with $\lim _{z \rightarrow \infty} g(z)=\infty$ in Table 2 which are closely related with the life distributions. As discussed in Section, the Exponential distribution arises as a limiting distribution for the Lomax distribution when the shape parameter $\alpha$ approaches infinity. However, these models in Table 2 do not satisfy the restriction on $g$ to be strictly positive with a finite limit at infinity as in condition (1). However, these are examples of the choices of $g(z)$ satisfying $\lim _{z \rightarrow \infty} g(z)=\infty$ which corresponds to some popular distributions as the particular cases. Table 2 displays the well-known Exponential, Rayleigh, Weibull, Gompertz, and Benini distribution as the member of an extended GLM family of distributions satisfying $\lim _{z \rightarrow \infty} g(z)=\infty$. In contrast to the GLM family of distributions, the distributions in the extended GLM family are light-tail distributions that belong to the basin of attraction of the Gumbel family of extreme value distributions, for example, Exponential, Rayleigh, Weibull, etc. For this extended GLM family of distributions, the survival distribution function decreases at an exponential rate. 

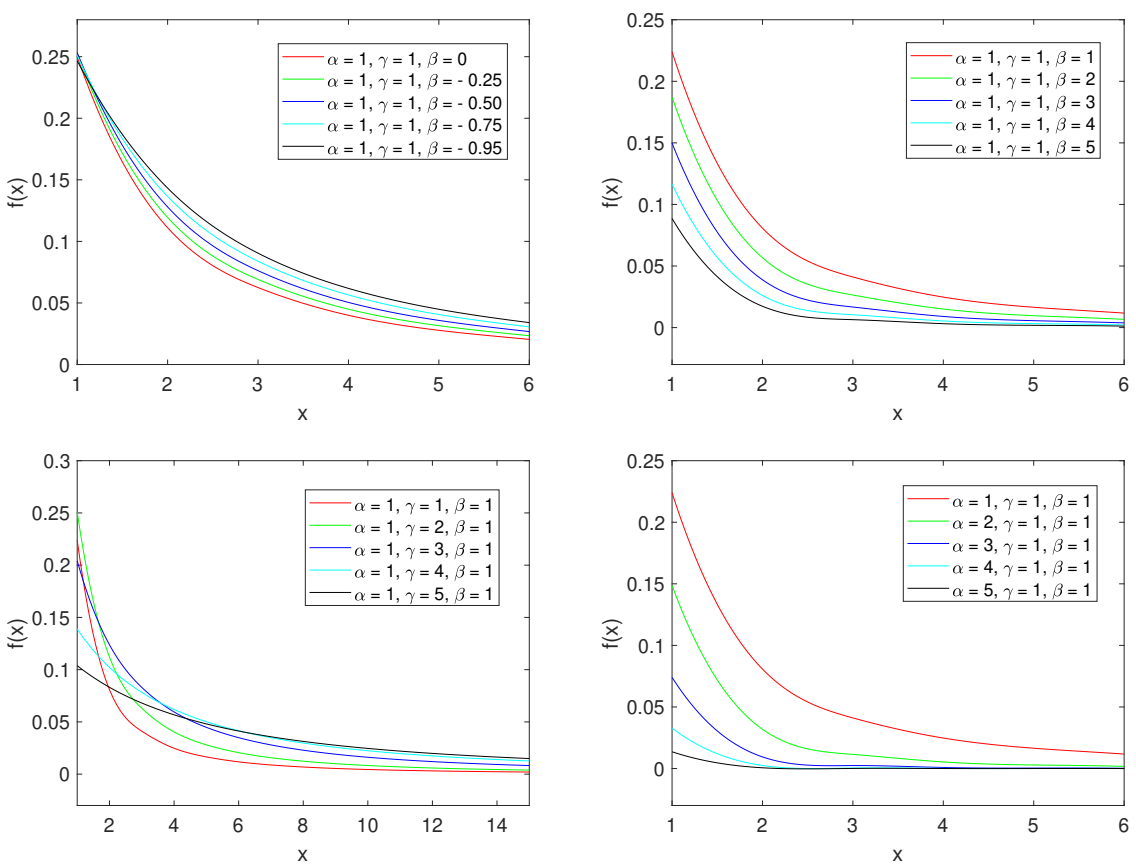

Fig 2. Plots of PDFs of the GLM Type-I distribution for different values of $\alpha, \beta$ and $\gamma$.
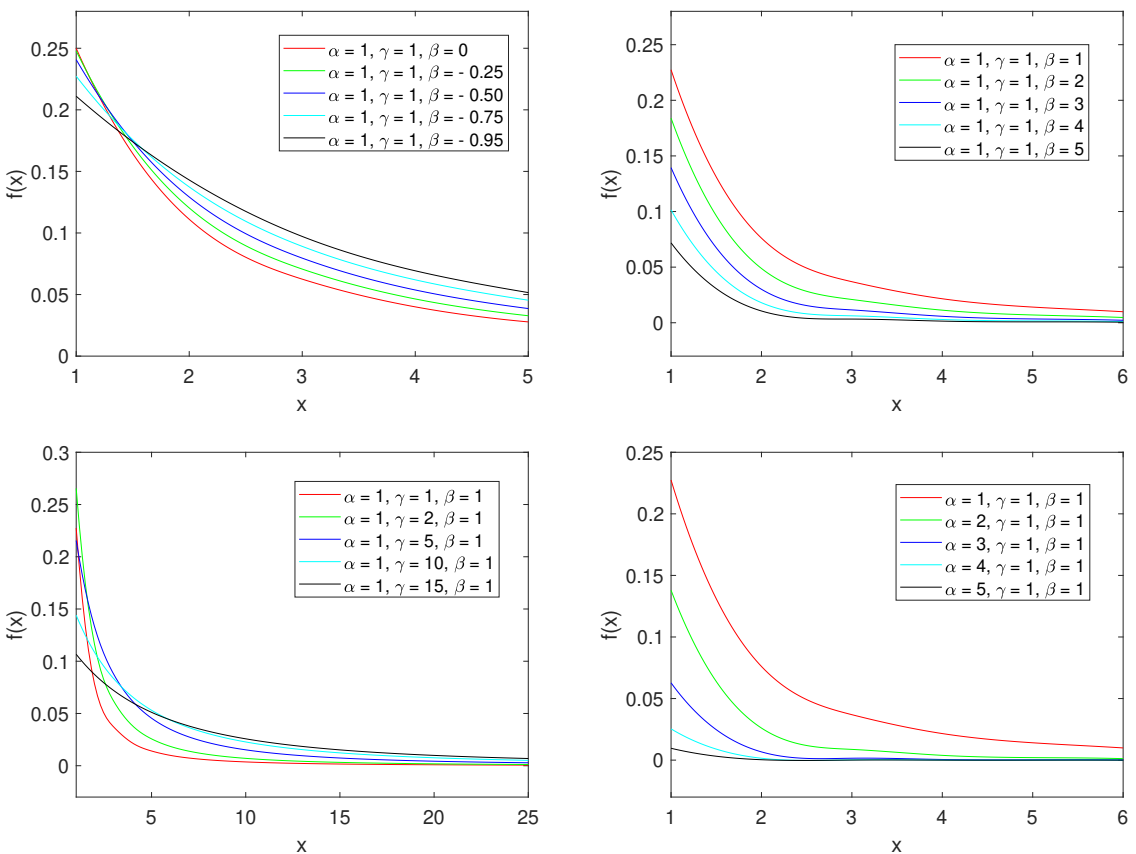

Fig 3. Plots of PDFs of the GLM Type-II distribution for different values of $\alpha, \beta$ and $\gamma$. 

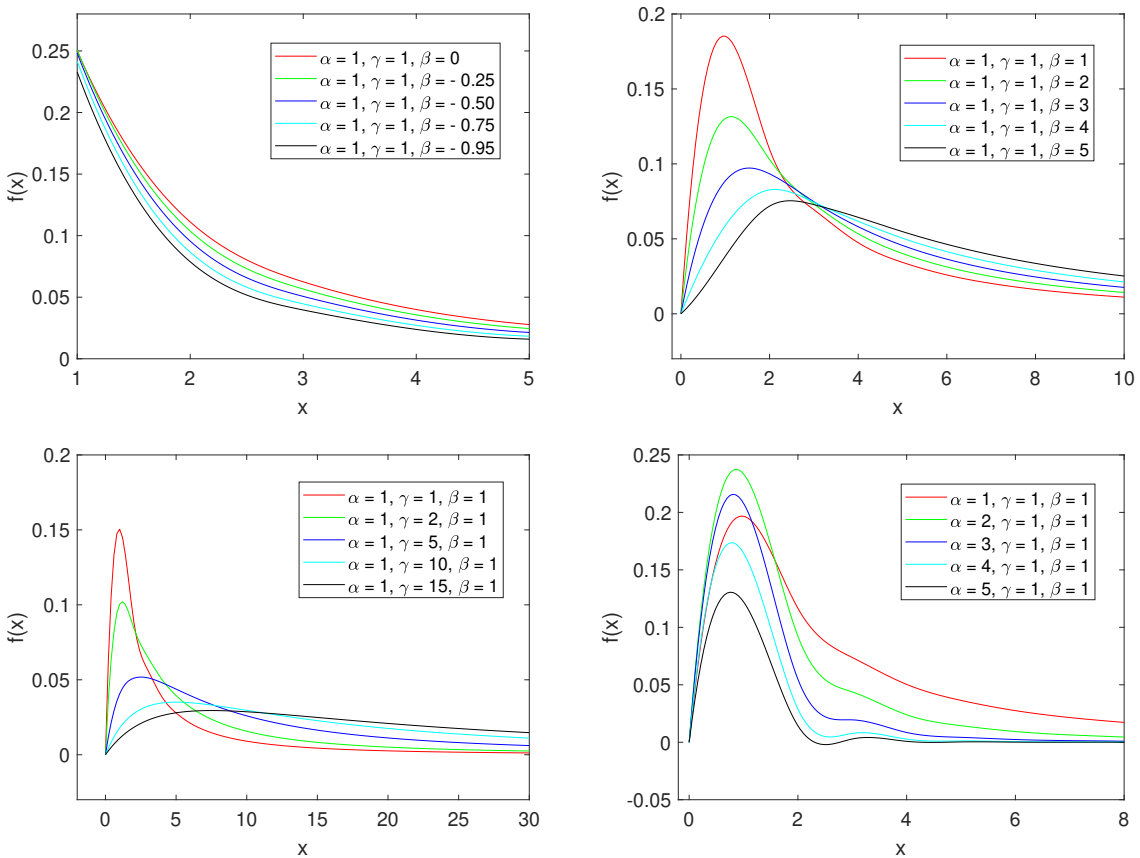

Fig 4. Plots of PDFs of the GLM Type-III distribution for different values of $\alpha, \beta$ and $\gamma$.
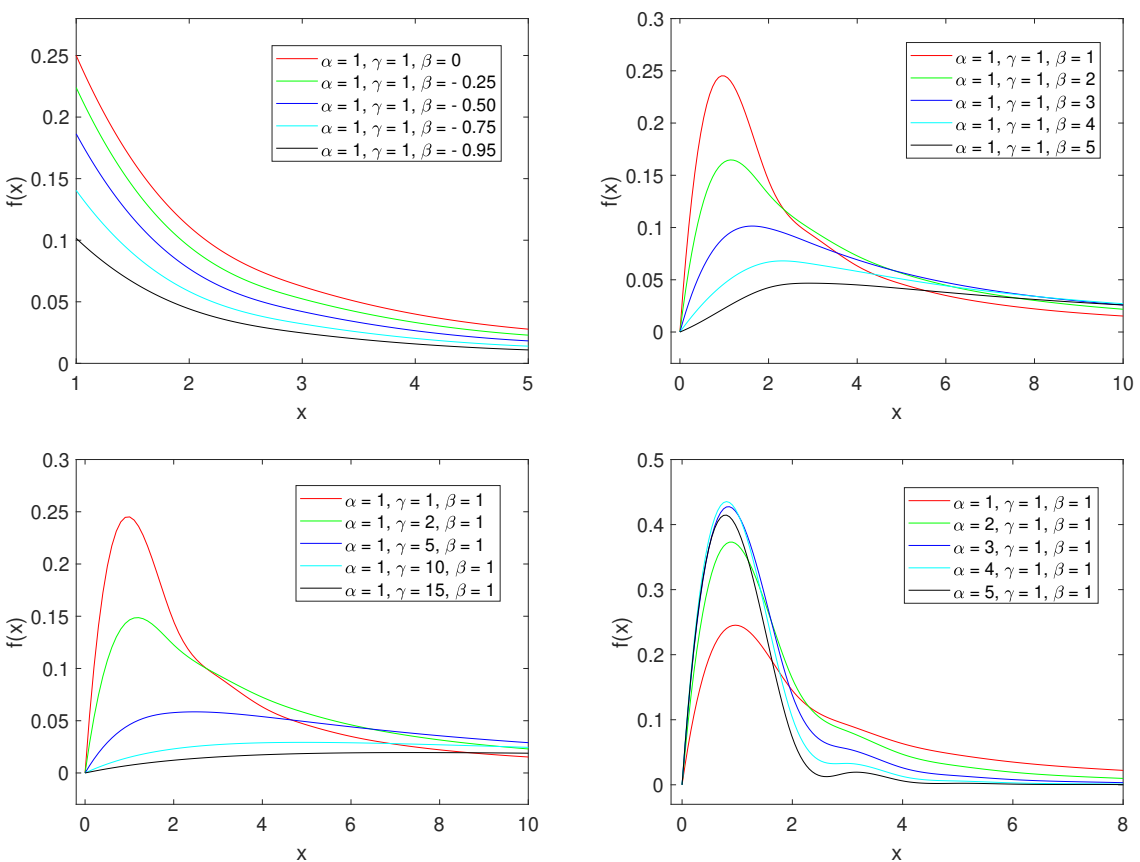

Fig 5. Plots of PDFs of the GLM Type-IV distribution for different values of $\alpha, \beta$ and $\gamma$. 
Table 2. Other extended family of GLM distributions with $\lim _{z \rightarrow \infty} g(z)=\infty$.

\begin{tabular}{|c|c|c|c|}
\hline$g(z), \forall z>0$ & $\beta$ & Distribution & $f(x), \forall x>0$ \\
\hline$\frac{z}{\log (1+z)}$ & & Exponential & $\frac{1}{\gamma} \exp \left[-\frac{x}{\gamma}\right]$ \\
\hline$\frac{z^{2}}{2 \log (1+z)}$ & & Rayleigh & $\frac{x}{\gamma^{2}} \exp \left[-\frac{x^{2}}{\left(2 \gamma^{2}\right)}\right]$ \\
\hline$\frac{z^{\beta}}{\log (1+z)}$ & $\beta>0$ & Weibull & $\frac{\beta}{\gamma}\left(\frac{x}{\gamma}\right)^{\beta-1} \exp \left[-\left(\frac{x}{\gamma}\right)^{\beta}\right]$ \\
\hline$\frac{\beta\left(e^{z}-1\right)}{\log (1+z)}$ & $\beta>0$ & Gompertz & $\frac{\beta}{\gamma} e^{\frac{x}{\gamma}} \exp \left[-\beta\left(e^{\frac{x}{\gamma}}-1\right)\right]$ \\
\hline$\alpha[1+\beta \log (1+z)]$ & $\beta \geq 0$ & Benini & $\frac{\alpha}{\gamma}\left[1+\frac{x}{\gamma}\right]^{-(\alpha+1)}\left[1+2 \beta \log \left(1+\frac{x}{\gamma}\right)\right] \exp \left[-\alpha \beta \log ^{2}\left(1+\frac{x}{\gamma}\right)\right]$ \\
\hline
\end{tabular}

\section{Statistical properties of the GLM family}

We study several extreme value properties of the new family of standard GLM 192 distributions from the perspective of extreme value and risk theory [37. Also, we 193 discuss the parameter estimation and goodness of fit for the proposed generalized 194 Lomax models.

\section{Extreme Value Properties}

Theorem 1. The general CDF (2) of the GLM family of distributions satisfying (1) ${ }_{197}$

(a) is heavy-tailed.

(b) is right-tail equivalent to Pareto distribution.

(c) belongs to dominated-variation distributions.

(d) belongs to the family of long-tailed distributions.

(e) belongs to the maximum domain of attraction (MDA) of the Frechet distribution.

Proof. (a) Any probability distribution function $F$ is called heavy-tail if it satisfies the following:

$$
\lim _{x \rightarrow \infty} \exp \{\lambda x\}(1-F(x))=\infty, \text { for any } \lambda>0 .
$$

Thus, for the GLM family of distributions, we observe that

$$
\lim _{x \rightarrow \infty} \exp \{\lambda x\}(1-F(x))=\lim _{x \rightarrow \infty} \exp \left[\lambda x-g\left(\frac{x}{\gamma}\right) \log \left(1+\frac{x}{\gamma}\right)\right]=\infty
$$

where $\lambda, \gamma>0$.

(b) To show the tail-equivalent property, we compute

$$
\lim _{x \rightarrow \infty} \frac{1-F(x)}{1-G(x)}=\lim _{x \rightarrow \infty} \frac{\exp \left[-g\left(\frac{x}{\gamma}\right) \log \left(1+\frac{x}{\gamma}\right)\right]}{\exp \left[-\alpha \log \left(1+\frac{x}{\gamma}\right)\right]}=1, \forall \gamma>0
$$

where $G(x)$ is the CDF of the Pareto Type-II distribution.

(c) Any probability distribution function $F$ is said to belong to the class dominated-variation distributions if

$$
\limsup _{x \rightarrow \infty} \frac{1-F(x)}{1-F(2 x)}<\infty .
$$


Thus, for the GLM family of distributions, $\lim _{x \rightarrow \infty} \frac{1-F(x)}{1-F(2 x)}=\lim _{x \rightarrow \infty} \exp \left[g\left(\frac{x}{\gamma}\right) \log \left(\frac{1+\frac{2 x}{\gamma}}{1+\frac{x}{\gamma}}\right)\right]$

$$
\begin{aligned}
& =\exp (\alpha \log 2) \\
& =2^{\alpha}<\infty
\end{aligned}
$$

where $\alpha>0$.

(d) Any probability distribution function $F$ is called long-tailed distributions if $F$ has (right) unbounded support and for any fixed $k>0$

$$
\lim _{x \rightarrow \infty} \frac{1-F(x+k)}{1-F(x)}=1 .
$$

Thus, $\lim _{x \rightarrow \infty} \frac{1-F(x+k)}{1-F(x)}=\lim _{x \rightarrow \infty} \exp \left[-g\left(\frac{x}{\gamma}\right) \log \left(1+\frac{(k / \gamma)}{\left(1+\frac{x}{\gamma}\right)}\right)\right]=1$,

where $\gamma>0$

(e) It should be noted that any function $g$ as defined in Section satisfying $\lim _{z \rightarrow \infty} g(z)=\alpha>0$, is slowly varying at infinity:

$$
\begin{aligned}
& \lim _{z \rightarrow \infty} \frac{g(t z)}{g(z)}=1, \forall t>0 . \\
& \text { Now, } \lim _{x \rightarrow \infty} \frac{1-F(t x)}{1-F(x)}=\lim _{x \rightarrow \infty} \frac{\left(1+\frac{t x}{\gamma}\right)^{-g\left(\frac{t x}{\gamma}\right)}}{\left(1+\frac{x}{\gamma}\right)^{-g\left(\frac{x}{\gamma}\right)}} \\
& =t^{-\alpha}, \forall t>0 \text { and } \gamma>0 .
\end{aligned}
$$

Thus, GLM family of distributions comprise of continuously changing distributions at $\quad 207$ infinity and belong to the MDA of the Frechet distribution.

\section{Parameter estimation}

Let $x_{1}, x_{2}, \ldots, x_{n}$ be a sample of size $n$ from standard $\operatorname{GLM}(\alpha, \beta, \gamma)$ distributions (GLM Type-I, Type-II, Type-III and Type-IV) as reported in Table 1 . We present the procedure for parameter estimation including the log-likelihood functions and corresponding normal equations only for a particular GLM model, namely GLM Type-IV distribution. Similar derivations can be obtained for the other three GLM Type distributions.

The $\log$-likelihood function $\ell \equiv \ell(x ; \alpha, \beta, \gamma)$ for the vector of parameters $\Theta=(\alpha, \beta, \gamma)^{T}$ corresponding to GLM Type-IV distributions is given by

$$
\begin{aligned}
\ell & =n \log (\alpha)-\sum_{i=1}^{n} \log \left(\gamma+x_{i}\right)+\sum_{i=1}^{n} \log \left[\beta+1+w_{i}\right] \\
& +\beta \sum_{i=1}^{n} \log \left[w_{i}\right]-(\beta+1) \sum_{i=1}^{n} \log \left[1+w_{i}\right]-\alpha \sum_{i=1}^{n} \frac{\left[w_{i}\right]^{\beta+1}}{\left[1+w_{i}\right]^{\beta}}
\end{aligned}
$$

where $n$ is the sample size and $w_{i}=\log \left(1+\frac{x_{i}}{\gamma}\right)$. The maximum likelihood estimates of $\quad{ }^{219}$ the unknown parameter vector $(\alpha, \beta, \gamma)$ are those that maximizes the log-likelihood $\quad{ }_{220}$ function $\ell$ in Eqn. (4). 
The normal equations can be obtained by taking the partial derivatives of Eqn. (4) ${ }_{222}$ w.r.t. $\alpha, \beta, \gamma$ and equating them to zero as follows.

$$
\begin{gathered}
\frac{\partial \ell}{\partial \alpha}=\frac{n}{\alpha}-\sum_{i=1}^{n} \frac{w_{i}^{\beta+1}}{\left(1+w_{i}\right)^{\beta}} \\
\frac{\partial \ell}{\partial \beta}=\sum_{i=1}^{n} \frac{1}{\left(1+\beta+w_{i}\right)}+\sum_{i=1}^{n} \log \left(\frac{w_{i}}{1+w_{i}}\right) \times\left[1-\frac{\alpha w_{i}^{\beta+1}}{\left(1+w_{i}\right)^{\beta}}\right] \\
\frac{\partial \ell}{\partial \gamma}=-\sum_{i=1}^{n} \frac{1}{\left(\gamma+x_{i}\right)}+\sum_{i=1}^{n} \frac{x_{i}}{\gamma\left(\gamma+x_{i}\right)} \times\left\{\frac{\beta+1}{\left(1+w_{i}\right)}-\frac{\beta}{w_{i}}-\frac{1}{\left(1+\beta+w_{i}\right)}\right\} \\
+\alpha \sum_{i=1}^{n} \frac{x_{i}}{\gamma\left(\gamma+x_{i}\right)}\left[\frac{\left(1+\beta+w_{i}\right) w_{i}^{\beta}}{\left(1+w_{i}\right)^{\beta+1}}\right]
\end{gathered}
$$

The MLEs of the three parameters for the GLM Type-IV distributions with $\alpha, \beta$, and $\gamma$ can be obtained by setting the above partial derivatives to zero and then solving them simultaneously. Eqns. (5), (6) and (7) does not have closed-form solutions, thus, an iterative algorithm can be adapted to solve these equations numerically. For practical implementation of the model, we fit the standard GLM models in the whole range of the data sets with quasi-Newton BFGS numerical algorithm with initial values to be chosen as $\left(\hat{\alpha_{0}}, \hat{\beta_{0}}, \hat{\gamma_{0}}\right)=(1,0,1)$ to find the MLE estimates of the parameters.

\section{Goodness of fit}

How well the large-scale real-world complex network data sets correspond to the fitted (hypothesized) standard GLM family of distributions can be determined by the goodness-of-fit test. To this end, we have derived the $p$-value corresponding to the Chi-square statistic test, which will evaluate the goodness-of-fit for the GLM Type-I, Type-II, Type-III, and Type-IV distributions. We obtain the $p$-values using bootstrap resampling computational technique as follows: First, we decide the best fit of the proposed GLM family of distributions corresponding to the data after estimating the parameters using MLE and then evaluate the $p$-values through Chi-square statistic test for the goodness-of-fit of the best-fitted GLM model for the data. Then we generate 50000 synthetic network data sets from the concerned GLM distribution and calculate the Chi-square statistic ( $p$-values) for each of the generated synthetic data sets. Finally, we obtain the $p$-value for the generated synthetic data sets as the fraction of GLM synthetic data sets with a Chi-square value greater than the empirical one. Higher $p$-values signify that the proposed model is 'most' suitable and effective for the data set. Furthermore, we compute few other standard statistical measures viz. KLD, RMSE, and MAE for quantifying and comparing the goodness-of-fit for the proposed standard GLM family of distributions to the other commonly used heavy-tailed distributions for modeling various heavy-tailed real-world complex networks.

\section{Real-world Applications}

\section{Description of data sets}

We consider large scale network data sets from different disciplines, namely social networks, collaboration networks, web graphs, citation networks, biological networks, product co-purchasing networks, temporal networks, communication networks, ground-truth networks, and brain networks. We study several individual data sets from each discipline. These data sets are publicly available at 
Table 3. Characteristics and statistical measures for fifty real-world complex network data sets

\begin{tabular}{|c|c|c|c|c|c|c|}
\hline \multicolumn{2}{|r|}{$\begin{array}{l}\text { Data } \\
\text { sets }\end{array}$} & \multirow[t]{2}{*}{$\begin{array}{l}\text { No. of } \\
\text { Nodes }\end{array}$} & \multirow[t]{2}{*}{$\begin{array}{l}\text { No. of } \\
\text { Edges }\end{array}$} & \multicolumn{3}{|c|}{ Statistical Measures } \\
\hline & & & & s.d. & mean & $C V$ \\
\hline \multirow{11}{*}{$\begin{array}{c}\text { Social } \\
\text { Networks }\end{array}$} & ego-Twitter(In) & 81,306 & $1,768,149$ & 57.965 & 21.747 & 2.6654 \\
\hline & ego-Gplus(In) & 107,614 & $13,673,453$ & 1404.8 & 283.42 & 4.9568 \\
\hline & soc-Slashdot & 70,068 & 358,647 & 35.069 & 10.237 & 3.4260 \\
\hline & soc-Delicious(In) & 536,108 & $1,365,961$ & 39.826 & 10.673 & 3.7312 \\
\hline & soc-Digg(In) & 770,799 & $5,907,132$ & 166.61 & 46.584 & 3.5765 \\
\hline & soc-Academia & 200,169 & $1,398,063$ & 48.297 & 14.259 & 3.3871 \\
\hline & LiveJournal(In) & $4,847,571$ & $68,993,773$ & 44.969 & 15.368 & 2.9261 \\
\hline & Dogster-Friendship & 426,821 & $8,546,581$ & 284.06 & 40.033 & 7.0950 \\
\hline & Higgs-Twitter(In) & 456,626 & $14,855,842$ & 350.91 & 54.786 & 6.4051 \\
\hline & Artist-Facebook & 50,615 & 819,307 & 63.427 & 32.366 & 1.9596 \\
\hline & Athletes-Facebook & 13,866 & 86,859 & 17.978 & 12.438 & 1.4453 \\
\hline Citation & cit-HepTh(In) & 27,770 & 352,807 & 43.139 & 15.220 & 2.8342 \\
\hline \multirow[t]{3}{*}{ Networks } & cit-HepPh(In) & 34,546 & 421,578 & 27.286 & 14.933 & 1.8271 \\
\hline & cit-Patents(In) & $3,774,768$ & $16,518,948$ & 6.9125 & 5.0687 & 1.3637 \\
\hline & cit-Citeseer(In) & 227,320 & 814,134 & 9.8260 & 5.4322 & 1.8088 \\
\hline \multirow{5}{*}{$\begin{array}{l}\text { Collaboration } \\
\text { Networks }\end{array}$} & ca-CondMat & 23,133 & 93,497 & 10.671 & 8.0189 & 1.3308 \\
\hline & ca-AstroPh & 18,772 & 198,110 & 30.568 & 21.103 & 1.4484 \\
\hline & ca-GrQc & 5,242 & 14,496 & 7.9186 & 5.5284 & 1.4322 \\
\hline & ca-HepPh & 12,008 & 118,521 & 46.654 & 19.696 & 2.3687 \\
\hline & ca-HepTh & 9,877 & 25,998 & 6.1867 & 5.2618 & 1.1757 \\
\hline \multirow{5}{*}{$\begin{array}{l}\text { Web } \\
\text { Graphs }\end{array}$} & Google(In) & 875,713 & $5,105,039$ & 43.320 & 7.1444 & 6.0634 \\
\hline & BerkStan(In) & 685,230 & $7,600,595$ & 300.08 & 12.316 & 24.364 \\
\hline & Wikipedia2009(In) & $1,864,433$ & $4,507,315$ & 12.846 & 4.8903 & 2.6268 \\
\hline & WikipediaLinkFr(In) & $4,906,478$ & $113,122,279$ & 1864.4 & 48.608 & 38.356 \\
\hline & Hudong(In) & $1,984,484$ & $14,869,483$ & 199.28 & 16.467 & 12.101 \\
\hline \multirow{5}{*}{$\begin{array}{l}\text { Biological } \\
\text { Networks }\end{array}$} & Yeast-PPIN & 2,361 & 7,182 & 8.0800 & 6.0838 & 1.3281 \\
\hline & Diseasome & 3,926 & 7,823 & 9.1009 & 5.5334 & 1.6447 \\
\hline & Bio-Mouse-Gene & 45,101 & $14,506,199$ & 856.67 & 643.27 & 1.3317 \\
\hline & Bio-Dmela & 7,393 & 25569 & 10.782 & 6.9170 & 1.5587 \\
\hline & Bio-WormNet-v3 & 16,347 & 762,822 & 138.17 & 93.328 & 1.4805 \\
\hline \multirow{3}{*}{$\begin{array}{c}\text { Product } \\
\text { co-purchasing } \\
\text { networks }\end{array}$} & amazon0601(In) & 403,394 & $3,387,388$ & 15.279 & 8.3989 & 1.8191 \\
\hline & amazon0505(In) & 410,236 & $3,356,828$ & 15.313 & 8.1826 & 1.8714 \\
\hline & amazon0312(In) & 400,727 & $3,200,444$ & 15.073 & 7.9865 & 1.8873 \\
\hline Temporal & sx-mathoverflow(In) & 24,818 & 506,550 & 31.476 & 10.424 & 3.0195 \\
\hline \multirow[t]{3}{*}{ Networks } & sx-stackoverflow(In) & $2,601,977$ & $63,497,050$ & 186.00 & 27.647 & 6.7278 \\
\hline & sx-superuser(In) & 194,085 & $1,443,339$ & 23.782 & 5.8239 & 4.0836 \\
\hline & sx-askubuntu (In) & 159,316 & 964,437 & 18.404 & 4.3856 & 4.1966 \\
\hline \multirow{3}{*}{$\begin{array}{c}\text { Communication } \\
\text { Networks }\end{array}$} & Email-Enron & 36,692 & 183,831 & 36.100 & 10.021 & 3.6027 \\
\hline & Wiki-Talk(In) & $2,394,385$ & $5,021,410$ & 12.259 & 2.1195 & 5.7844 \\
\hline & Rec-Libimseti(In) & 220,970 & $17,359,346$ & 413.71 & 102.85 & 4.0227 \\
\hline \multirow{5}{*}{$\begin{array}{l}\text { Ground-truth } \\
\text { Networks }\end{array}$} & Wiki-Topcats & $1,791,489$ & $28,511,807$ & 283.78 & 15.915 & 17.831 \\
\hline & com-Friendster & $65,608,366$ & $1,806,067,135$ & 137.81 & 55.056 & 2.5031 \\
\hline & com-LiveJournal & $3,997,962$ & $34,681,189$ & 42.957 & 17.349 & 2.4759 \\
\hline & com-Orkut & $3,072,441$ & $117,185,083$ & 154.78 & 76.281 & 2.0291 \\
\hline & com-Youtube & $1,134,890$ & $2,987,624$ & 50.754 & 5.2650 & 9.6398 \\
\hline Brain & Human25890-session1 & 177,584 & $15,669,036$ & 319.01 & 176.47 & 1.8078 \\
\hline \multirow[t]{4}{*}{ Networks } & Human25890-session 2 & 723,881 & $158,147,409$ & 667.91 & 436.94 & 1.5286 \\
\hline & Human25864-session2 & 692,957 & $133,727,516$ & 554.48 & 385.96 & 1.4366 \\
\hline & Human25913-session2 & 726,197 & $183,978,766$ & 446.92 & 258.99 & 1.7256 \\
\hline & Human25886-session1 & 780,185 & $158,184,747$ & 558.41 & 405.50 & 1.3771 \\
\hline
\end{tabular}


http://snap.stanford.edu/data/index.html. These are the most standard network data sets that have heavy-tailed behaviors and are used for modeling in the statistical paradigm 10, 25, 26. Previous studies focused on using standard single statistical distributions, namely power-law, Lomax (Pareto Type-II), Exponential, Log-normal for modeling this wide variety of network data sets $[1,11$. But these models fail in capturing the lower-degree nodes while modeling the degree distributions. To overcome the drawback, we consider a new family of the proposed GLM family of distributions. Note that the proposed family of heavy-tailed GLM distributions can model these large-scale network data sets in the whole range. An overview of these publicly available network data sets is presented in Table 3. Some statistical measures, for example, mean, standard deviation (s.d.), and calculated $C V$ corresponding to the degree distributions of each network data set, are also given in Table 3 . It is important to note that the empirical $C V$ for all the data sets is greater than one, as reported in Table 3 .

This section shows the effectiveness of the proposed GLM family of distributions by comparing it with six other established, well-known models, such as Lomax, power-law, power-law with cutoff, Log-normal, and Exponential distributions. The performances of these commonly used heavy-tailed and life distribution models are reported in Table 6 . Finally, we test the adequacy of the GLM family of distributions compared to these heavy-tailed and light-tailed distributions over fifty large-scale real-world network data sets in the whole range. To do the numerical computations for fitting the new family of GLM distributions in the whole range for large-scale real-world network data sets, we use the R statistical software function 'optim'. The 'optim' function makes use of the limited memory quasi-Newton L-BFGS-B optimization algorithm in the background by taking the initial parameter values as $\left(\hat{\alpha_{0}}, \hat{\beta_{0}}, \hat{\gamma_{0}}\right)=(1,0,1)$.

We fitted the GLM Type-I, GLM Type-II, GLM Type-III, and GLM Type-IV models over the entire degree distribution of the complex network data sets by applying MLE to estimate the parameters, as discussed in Section and. We measure the goodness-of-fit of the proposed GLM distributions through the bootstrap resampling Chi-square test. Table 4 represents the estimated values of the parameters for four newly introduced GLM models along with the $p$-values. It is observed that the obtained $p$-values $>0.4$ for all the cases. Also, it is clear that the MLE estimated parameter values for all the network data sets satisfy the condition $\alpha>0, \beta>-1$, and $\gamma>0$. The empirical analysis shows that the proposed standard GLM distributions fit well in the majority of the network data sets under consideration. We provide enough statistical evidence of fitting the proposed GLM models in Table 5. We leverage some popular statistical measures, viz., MAE, RMSE, and KLD, to evaluate and compare the performance of the proposed standard GLM distributions with others as shown in Table 5.

The overall performance of the proposed GLM models are better in terms of RMSE, MAE, and KLD values for most of the networks compared to other competing distributions, which suggests that the proposed GLM family plausibly fits the observed node distribution. Empirical data analysis suggests that the parameter estimation yield the values for specifying the best fit of the proposed models. However, the estimated values alone do not give any effective information regarding the validity or the goodness-of-fit of the underlying models. From Table 5 and 6 , it can be concluded that the GLM Type-I model outperforms all the competitive models for four data sets. In contrast, the GLM Type-III model outperforms others for eight network data sets from various domains. The GLM Type-II model performs superior to others for thirteen data sets. The power-law model with exponential cutoff [11] performed better than the GLM models for four out of fifty real-world complex heavy-tailed network data sets. Overall, the performance of the GLM Type-IV model is very consistent across all the data sets,

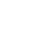

2

4

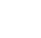

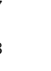

0


Table 4. Parameter estimates for the GLM models over 50 different real-world network data

\begin{tabular}{|c|c|c|c|c|c|c|c|c|c|c|c|c|c|c|c|c|}
\hline \multirow[t]{2}{*}{ Data } & \multicolumn{4}{|c|}{ GLM Type-I } & \multicolumn{4}{|c|}{ GLM Type-II } & \multicolumn{4}{|c|}{ GLM Type-III } & \multicolumn{4}{|c|}{ GLM Type-IV } \\
\hline & $\hat{\alpha}$ & $\hat{\beta}$ & $\widehat{\gamma}$ & $p$ & $\hat{\alpha}$ & $\widehat{\beta}$ & $\widehat{\gamma}$ & $p$ & $\widehat{\alpha}$ & $\widehat{\beta}$ & $\hat{\gamma}$ & $p$ & $\widehat{\alpha}$ & $\widehat{\beta}$ & $\hat{\gamma}$ & $p$ \\
\hline ego-Twitter(In) & 1.4425 & 0.4908 & 16.404 & 0.9600 & 1.4267 & -0.0018 & 11.098 & 0.9789 & 1.9244 & 0.4791 & 35.361 & 0.8746 & 1.9922 & -0.3591 & 30.543 & 0.9920 \\
\hline ego-Gplus(In) & 0.6257 & 0.4903 & 15.236 & 0.9540 & 0.6283 & -0.0015 & 10.577 & 0.9217 & 0.6666 & -0.5953 & 8.8974 & 0.8792 & 0.7108 & -0.4983 & 23.077 & 0.9963 \\
\hline soc-Slashdot & 0.8463 & 0.1220 & 0.8679 & 0.9515 & 0.8478 & -0.0047 & 0.7968 & 0.9746 & 0.8684 & -0.5504 & 0.5696 & 0.9825 & 0.8663 & -0.6228 & 1.0461 & 0.9955 \\
\hline soc-Delicious(In) & 1.0804 & 0.5820 & 3.4346 & 0.9782 & 1.0853 & -0.0001 & 2.2622 & 0.9638 & 1.0904 & -0.3791 & 1.7701 & 0.9600 & 1.3630 & -0.6819 & 5.3709 & 0.9960 \\
\hline soc-Digg(In) & 0.7204 & 0.4587 & 4.3544 & 0.9810 & 0.7235 & -0.0025 & 3.1117 & 0.9573 & 0.7544 & -0.5379 & 2.4772 & 0.9271 & 0.7931 & -0.6928 & 5.5163 & 0.9890 \\
\hline soc-Academia & 1.7382 & 0.4769 & 15.286 & 0.5872 & 1.7182 & -0.0229 & 10.202 & 0.6480 & 2.6546 & 0.2978 & 38.846 & 0.5901 & 2.7429 & -0.3737 & 36.644 & 0.6087 \\
\hline LiveJournal(In) & 1.1060 & 0.5473 & 6.4094 & 0.8713 & 1.1016 & -0.0010 & 4.2266 & 0.8010 & 1.0846 & -0.3344 & 3.3178 & 0.8245 & 2.6892 & -0.7272 & 51.933 & 0.8983 \\
\hline Dogster-Friendship & 1.5772 & -0.6461 & 11.177 & 0.9178 & 1.5188 & -0.7341 & 8.0293 & 0.9200 & 1.5493 & -0.6744 & 9.5169 & 0.9246 & 1.5634 & 0.3108 & 14.057 & 0.9500 \\
\hline Higgs-Twitter(In) & 1.6379 & 0.7828 & 8.8420 & 0.9457 & 1.6437 & -0.0010 & 33.501 & 0.9652 & 1.0009 & 3.5570 & 11.578 & 0.9700 & 1.6797 & -0.0347 & 36.204 & 0.9870 \\
\hline Artist-Facebook & 1.7616 & 0.4736 & 38.243 & 0.9871 & 1.7268 & 0.0024 & 25.915 & 0.9889 & 1.0003 & 2.2910 & 24.774 & 0.9677 & 2.0117 & -0.1445 & 39.337 & 0.9812 \\
\hline Athletes-Facebook & 3.0346 & -0.4698 & 15.827 & 0.9023 & 2.9388 & -0.5970 & 11.569 & 0.9230 & 3.1509 & -0.0843 & 21.497 & 0.9450 & 3.1229 & 0.1406 & 21.180 & 0.9640 \\
\hline cit-HepTh(In) & 1.5176 & 0.5016 & 12.196 & 0.8020 & 1.5010 & 0.0016 & 8.2280 & 0.8622 & 1.7932 & 0.4842 & 19.284 & 0.8238 & 1.8410 & -0.3093 & 16.416 & 0.8730 \\
\hline cit-HepPh(In) & 1.6478 & 0.4899 & 15.227 & 0.9800 & 1.6216 & $8.8 \mathrm{e}-04$ & 10.267 & 0.9823 & 2.4380 & 0.3225 & 35.806 & 0.9730 & 2.5553 & -0.3622 & 34.349 & 0.9900 \\
\hline cit-Patents(In) & 3.0822 & 0.4155 & 12.407 & 0.7600 & 2.9957 & 0.0013 & 8.6685 & 0.7829 & 4.3878 & 0.1154 & 20.912 & 0.7760 & 4.4822 & -0.2534 & 21.689 & 0.8080 \\
\hline cit-Citeseer(In) & 1.9770 & 0.5650 & 6.8768 & 0.5620 & 1.9529 & -0.0012 & 4.4284 & 0.5930 & 2.2391 & 0.3025 & 8.3053 & 0.6210 & 2.2630 & -0.2788 & 7.4150 & 0.6350 \\
\hline ca-CondMat & 3.0282 & -0.7145 & 7.8751 & 0.9790 & 2.8862 & -0.7832 & 5.5961 & 0.9821 & 3.1195 & -0.2201 & 10.704 & 0.9328 & 3.1068 & 0.3615 & 10.535 & 0.9896 \\
\hline ca-AstroPh & 1.9271 & 0.4732 & 30.925 & 0.9537 & 1.8673 & -0.0010 & 20.590 & 0.9480 & 1.0040 & 28.012 & 6.4950 & 0.9729 & 16.434 & 17.276 & 9.0101 & 0.9990 \\
\hline $\mathrm{ca}-\mathrm{GrQc}$ & 1.6553 & -0.9564 & 3.5532 & 0.6982 & 1.4603 & -0.8822 & 4.4838 & 0.7290 & 1.5173 & -0.4341 & 1.6545 & 0.7628 & 2.2624 & 3.5861 & 0.6765 & 0.7849 \\
\hline $\mathrm{ca}-\mathrm{HepPh}$ & 0.8466 & -0.8552 & 2.9314 & 0.8092 & 0.7828 & -0.7766 & 2.7426 & 0.8009 & 0.6181 & 1.1938 & 3.6423 & 0.7985 & 0.9798 & 2.8780 & 0.6791 & 0.8163 \\
\hline ca-HерTh & 2.0930 & -0.2740 & 2.0444 & 0.8710 & 1.9578 & -0.9675 & 5.4783 & 0.8892 & 2.0088 & -0.9969 & 2.6700 & 0.9023 & 2.9417 & 5.2791 & 0.4825 & 0.9332 \\
\hline Google(In) & 1.0913 & 0.6327 & 1.9727 & 0.8938 & 1.0992 & 0.0007 & 1.2666 & 0.9012 & 1.1111 & -0.4390 & 0.9454 & 0.9520 & 1.1999 & -0.6399 & 2.0429 & 0.9780 \\
\hline $\operatorname{BerkStan}(\mathrm{In})$ & 1.4163 & -0.4362 & 5.1998 & 0.5010 & 1.1456 & -0.7842 & 8.0937 & 0.5541 & 1.1368 & -0.0213 & 3.1245 & 0.6020 & 1.4129 & 1.8449 & 0.7592 & 0.6250 \\
\hline Wikipedia2009(In) & 1.3230 & 0.5888 & 2.0563 & 0.8970 & 1.3377 & -0.0038 & 1.3722 & 0.9261 & 1.3513 & -0.3821 & 1.0539 & 0.9789 & 1.3988 & -0.6291 & 1.9658 & 0.9891 \\
\hline WikipediaLinkFr(In) & 0.7804 & 0.5529 & 4.3367 & 0.8800 & 0.7822 & 0.0021 & 2.8959 & 0.8128 & 0.8015 & -0.5066 & 2.2526 & 0.8735 & 1.0988 & -0.7123 & 9.8888 & 0.9152 \\
\hline Hudong(In) & 0.9796 & -0.2066 & 15.002 & 0.9200 & 0.7881 & 0.0085 & 0.3133 & 0.9374 & 0.7990 & -0.7314 & 0.1727 & 0.9720 & 1.1567 & 10.921 & 0.0013 & 0.9883 \\
\hline Yeast-PPIN & 1.4329 & 0.5402 & 5.2496 & 0.9680 & 1.4013 & 0.0015 & 3.4116 & 0.9718 & 1.2137 & 2.6648 & 7.8313 & 0.9870 & 10.535 & -0.4527 & 175.29 & 0.9930 \\
\hline Diseasome & 1.1069 & 0.6460 & 2.2879 & 0.7028 & 1.1030 & 0.0031 & 1.4262 & 0.7620 & 1.1031 & -0.3999 & 1.0749 & 0.7988 & 10.969 & -0.9493 & 134.52 & 0.8090 \\
\hline Bio-Mouse-Gene & 0.0579 & -0.7322 & 3.9518 & 0.9380 & 0.0512 & -0.5732 & 3.5602 & 0.9678 & 0.1190 & -0.9903 & 4.7784 & 0.9890 & $6.3 e-08$ & $-1.2 \mathrm{e}-02$ & $2.1 \mathrm{e}+00$ & 0.9898 \\
\hline Bio-Dmela & 1.5268 & 0.5971 & 6.1136 & 0.8972 & 1.5076 & 0.0003 & 3.8602 & 0.9342 & 3.5180 & 0.3183 & 39.215 & 0.9679 & 14.979 & -0.5053 & 498.27 & 0.9806 \\
\hline Bio-WormNet-v3 & 0.4182 & 0.5801 & 12.836 & 0.9780 & 0.4034 & -0.0002 & 7.9435 & 0.9890 & 0.5142 & -0.6171 & 9.3771 & 0.9829 & 5.6496 & -0.9801 & 704.71 & 0.9938 \\
\hline amazon0601(In) & 3.4448 & -0.6406 & 10.273 & 0.5026 & 3.2935 & -0.7309 & 7.3059 & 0.5567 & 3.5283 & -0.1446 & 14.262 & 0.5830 & 3.8261 & -0.7137 & 19.522 & 0.6010 \\
\hline amazon0505(In) & 3.4863 & -0.4779 & 11.883 & 0.5100 & 3.3632 & -0.6095 & 8.5613 & 0.5671 & 3.6248 & -0.0677 & 16.618 & 0.5892 & 3.8367 & -0.8006 & 19.984 & 0.6880 \\
\hline amazon0312(In) & 3.4280 & -0.5110 & 10.948 & 0.4997 & 3.3018 & -0.6336 & 7.8797 & 0.5102 & 3.5387 & -0.0850 & 15.171 & 0.5287 & 3.7631 & -0.8179 & 18.747 & 0.5890 \\
\hline sx-mathoverflow(In) & 1.3002 & -0.9831 & 4.2711 & 0.8751 & 1.0922 & -0.9254 & 5.2890 & 0.9127 & 1.1282 & -0.6887 & 6.4165 & 0.9457 & 1.4452 & 2.4236 & 0.8241 & 0.9846 \\
\hline sx-stackoverflow(In) & 1.0282 & -0.2710 & 3.8714 & 0.7982 & 1.0402 & -0.0038 & 4.8809 & 0.8086 & 1.0604 & -0.6329 & 3.1170 & 0.9780 & 1.0218 & -0.8224 & 4.4865 & 0.9490 \\
\hline sx-superuser(In) & 1.6803 & -0.8466 & 5.1872 & 0.9421 & 1.3392 & -0.6496 & 2.1189 & 0.9537 & 1.3425 & -0.4221 & 2.1865 & 0.9788 & 1.7401 & 2.1405 & 0.7284 & 0.9780 \\
\hline sx-askubuntu (In) & 2.0897 & -0.1210 & 6.1834 & 0.7002 & 1.6205 & -0.6537 & 7.0789 & 0.7546 & 1.5954 & -0.9139 & 4.1416 & 0.8249 & 2.1923 & 2.2069 & 0.7665 & 0.9300 \\
\hline Email-Enron & 1.2232 & -0.0338 & 2.5047 & 0.9045 & 1.2149 & -0.2599 & 1.9463 & 0.9246 & 1.0797 & 1.7058 & 3.4351 & 0.9550 & 1.2417 & -0.1275 & 2.9045 & 0.9641 \\
\hline Wiki-Talk(In) & 0.4360 & 18.634 & 2.2229 & 0.9452 & 1.5175 & 0.6688 & $3.2 \mathrm{e}-05$ & 0.8990 & 0.9895 & 59.520 & 0.0548 & 0.9782 & 1.5167 & -0.2846 & 0.0016 & 0.9900 \\
\hline Rec-Libimseti(In) & 0.5100 & 0.5417 & 5.2411 & 0.9247 & 0.5055 & 0.0004 & 3.3914 & 0.9354 & 0.5536 & -0.5814 & 3.2391 & 0.9556 & 2.5008 & -0.8496 & 331.18 & 0.9670 \\
\hline Wiki-Topcats & 1.1907 & -0.3884 & 2.3364 & 0.7400 & 1.1697 & -0.4969 & 1.8217 & 0.7893 & 1.1968 & -0.7570 & 1.6464 & 0.8420 & 1.1811 & 0.1998 & 2.6412 & 0.8310 \\
\hline com-Friendster & 0.4495 & 0.5330 & 2.1401 & 0.8080 & 0.4481 & -0.0014 & 1.4159 & 0.8123 & 0.4878 & -0.5807 & 1.2679 & 0.8450 & 4.5863 & -0.9188 & 590.01 & 0.9000 \\
\hline com-LiveJournal & 1.2144 & 0.5169 & 9.3591 & 0.6798 & 1.2065 & 0.0010 & 6.2874 & 0.7246 & 1.0222 & 30.033 & 26.596 & 0.7800 & 2.8206 & -0.6020 & 65.638 & 0.7980 \\
\hline com-Orkut & 3.2813 & -0.6192 & 94.848 & 0.9120 & 3.1372 & -0.7163 & 67.315 & 0.9570 & 0.9977 & 183.89 & 46.878 & 0.9544 & 3.7049 & 0.1292 & 167.93 & 0.9890 \\
\hline com-Youtube & 1.0835 & 0.4666 & 0.5561 & 0.7840 & 1.0994 & -0.0046 & 0.4157 & 0.8701 & 1.1142 & -0.5816 & 0.2706 & 0.8701 & 1.6113 & 8.3355 & 0.0094 & 0.8410 \\
\hline Human25890-session1 & 1.2418 & 0.5115 & 123.15 & 0.8701 & 1.2183 & 0.0025 & 81.378 & 0.8018 & 1.1098 & 1.8328 & 136.28 & 0.7812 & 1.6098 & -0.2076 & 168.75 & 0.8710 \\
\hline Human25890-session2 & 1.3134 & 0.4457 & 353.84 & 0.8990 & 1.2927 & 0.0019 & 246.67 & 0.9102 & 2.8936 & 0.2284 & 1711.6 & 0.9920 & 14.423 & -0.3466 & 18886.3 & 0.9980 \\
\hline Human25864-session2 & 0.2129 & -0.2582 & 5.2852 & 0.9781 & 1.2291 & $6.9 \mathrm{e}-04$ & 207.25 & 0.9631 & 0.2186 & -0.9777 & 4.7560 & 0.9800 & 16.250 & -0.3379 & 19217.8 & 0.9640 \\
\hline Human25913-session2 & 0.6684 & 0.5383 & 58.072 & 0.8403 & 0.6570 & -0.0006 & 37.681 & 0.8476 & 0.7088 & -0.5805 & 33.302 & 0.9257 & 7.1013 & -0.4681 & 5779.8 & 0.9290 \\
\hline Human25886-session1 & 1.5185 & 0.4334 & 430.83 & 0.8902 & 1.4983 & $8.4 \mathrm{e}-04$ & 303.87 & 0.8935 & 1.1350 & 2.4212 & 525.20 & 0.9456 & 21.591 & -0.3119 & 26975.9 & 0.9768 \\
\hline
\end{tabular}


Table 5. Performance measures of the proposed generalized Lomax models over 50 real world network data

\begin{tabular}{|c|c|c|c|c|c|c|c|c|c|c|c|c|c|}
\hline \multicolumn{2}{|c|}{$\begin{array}{l}\text { Data } \\
\text { sets }\end{array}$} & \multicolumn{3}{|c|}{ GLM Type-I } & \multicolumn{3}{|c|}{ GLM Type-II } & \multicolumn{3}{|c|}{ GLM Type-III } & \multicolumn{3}{|c|}{ GLM Type-IV (MLM [17] } \\
\hline $\begin{array}{l}\text { Social } \\
\text { Networks }\end{array}$ & 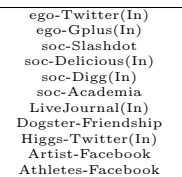 & $\begin{array}{l}27.161 \\
10.028 \\
30.607 \\
88.643 \\
23.306 \\
46.35 \\
1710.8 \\
28.237 \\
20.611 \\
10.710 \\
5.4833\end{array}$ & $\begin{array}{l}0.01264 \\
0.06337 \\
0.01406 \\
0.01255 \\
0.02362 \\
0.00932 \\
0.01968 \\
0.01245 \\
0.00787 \\
0.01147 \\
0.00899\end{array}$ & $\begin{array}{l}2.3209 \\
0.2927 \\
2.3965 \\
4.6171 \\
1.0020 \\
1.5352 \\
52.426 \\
0.7818 \\
0.4575 \\
2.3187 \\
1.4784\end{array}$ & $\begin{array}{l}29.325 \\
10.579 \\
30.581 \\
91.403 \\
24.568 \\
49.037 \\
1760.4 \\
27.864 \\
20.613 \\
11.861 \\
5.6072\end{array}$ & $\begin{array}{l}0.01354 \\
0.06444 \\
0.01407 \\
0.01326 \\
0.02391 \\
0.01020 \\
0.02112 \\
0.01215 \\
0.00793 \\
0.01193 \\
0.00901\end{array}$ & $\begin{array}{l}2.4709 \\
0.3034 \\
2.3944 \\
4.7889 \\
1.0214 \\
1.6258 \\
54.325 \\
0.7650 \\
0.4622 \\
2.4949 \\
1.4950\end{array}$ & $\begin{array}{l}17.465 \\
7.6188 \\
29.255 \\
87.087 \\
21.642 \\
16.758 \\
1647.4 \\
34.434 \\
20.2112 \\
8.4631 \\
4.2557\end{array}$ & $\begin{array}{l}0.00828 \\
0.06008 \\
0.01355 \\
0.01293 \\
0.02287 \\
0.00356 \\
0.02078 \\
0.01353 \\
0.00787 \\
0.01143 \\
\mathbf{0 . 0 0 8 7 6}\end{array}$ & $\begin{array}{l}1.3814 \\
0.2591 \\
2.3074 \\
4.6383 \\
0.9678 \\
0.5958 \\
52.644 \\
0.8853 \\
0.4639 \\
2.0212 \\
1.2988\end{array}$ & $\begin{array}{l}16.800 \\
1.6115 \\
31.527 \\
79.809 \\
13.634 \\
16.323 \\
243.99 \\
32.203 \\
19.821 \\
11.708 \\
4.4304\end{array}$ & $\begin{array}{l}0.00819 \\
0.05601 \\
0.01365 \\
0.00839 \\
0.02182 \\
0.00351 \\
6.13 e-04 \\
0.01328 \\
0.00785 \\
0.01079 \\
0.00879\end{array}$ & $\begin{array}{l}1.3498 \\
0.1825 \\
2.3951 \\
3.7730 \\
0.8440 \\
0.5705 \\
5.4026 \\
0.8502 \\
0.4710 \\
2.1381 \\
1.3252\end{array}$ \\
\hline $\begin{array}{l}\text { Citation } \\
\text { Networks }\end{array}$ & $\begin{array}{l}\text { cit-HepTh(In) } \\
\text { cit-HepPh(In) } \\
\text { cit-Patents(In) } \\
\text { cit-Citeseer(In) }\end{array}$ & $\begin{array}{l}7.0784 \\
18.816 \\
2400.3 \\
24.451\end{array}$ & $\begin{array}{l}0.01531 \\
0.01235 \\
0.00165 \\
0.00256\end{array}$ & $\begin{array}{l}0.7060 \\
2.8438 \\
213.79 \\
\mathbf{3 . 0 0 6 2}\end{array}$ & $\begin{array}{l}7.8479 \\
20.303 \\
2580.7 \\
27.533\end{array}$ & $\begin{array}{l}0.01584 \\
0.01311 \\
0.00192 \\
0.00278\end{array}$ & $\begin{array}{l}0.7536 \\
3.0223 \\
230.33 \\
3.3824\end{array}$ & $\begin{array}{l}3.3792 \\
10.051 \\
321.64 \\
41.668\end{array}$ & $\begin{array}{c}0.01358 \\
0.00834 \\
1.26 \mathrm{e}-04 \\
0.00231\end{array}$ & $\begin{array}{l}0.5150 \\
1.9528 \\
36.668 \\
3.8355\end{array}$ & $\begin{array}{l}3.2640 \\
\mathbf{9 . 6 8 1 0} \\
445.80 \\
40.728\end{array}$ & $\begin{array}{l}0.01354 \\
0.00821 \\
1.61 \mathrm{e}-04 \\
0.00228\end{array}$ & $\begin{array}{l}0.5071 \\
1.9016 \\
47.603 \\
3.3778\end{array}$ \\
\hline $\begin{array}{c}\text { Collaboration } \\
\text { Networks }\end{array}$ & $\begin{array}{l}\text { ca-CondMat } \\
\text { ca-AstroPh } \\
\text { ca-GrQc } \\
\text { ca-HepPh } \\
\text { ca-HepTh }\end{array}$ & $\begin{array}{l}13.146 \\
31.827 \\
10.147 \\
13.630 \\
24.676\end{array}$ & $\begin{array}{l}0.00471 \\
0.03335 \\
0.02796 \\
0.06938 \\
0.00943\end{array}$ & $\begin{array}{l}3.8859 \\
7.1574 \\
5.3808 \\
4.11517 \\
11.338\end{array}$ & $\begin{array}{l}\mathbf{1 2 . 9 1 4} \\
31.943 \\
\mathbf{9 . 9 1 3 1} \\
13.468 \\
25.222\end{array}$ & $\begin{array}{l}0.00471 \\
0.03425 \\
0.02801 \\
0.06945 \\
0.00955\end{array}$ & $\begin{array}{l}\mathbf{3 . 8 5 5 7} \\
7.2577 \\
\mathbf{5 . 2 7 2 4} \\
4.1297 \\
11.542\end{array}$ & $\begin{array}{l}15.609 \\
31.189 \\
10.069 \\
\mathbf{1 3 . 1 9 7} \\
25.066\end{array}$ & $\begin{array}{l}0.00483 \\
0.03423 \\
0.02800 \\
0.06814 \\
0.00949\end{array}$ & $\begin{array}{l}4.2729 \\
6.9837 \\
5.3306 \\
4.0911 \\
11.501\end{array}$ & $\begin{array}{l}14.830 \\
\mathbf{2 3 . 8 9 0} \\
15.850 \\
13.944 \\
\mathbf{2 3 . 2 8 0}\end{array}$ & $\begin{array}{l}0.00479 \\
0.02756 \\
0.03055 \\
0.06959 \\
\mathbf{0 . 0 0 8 9 6}\end{array}$ & $\begin{array}{l}4.1570 \\
5.9796 \\
7.2247 \\
4.1919 \\
10.851\end{array}$ \\
\hline $\begin{array}{c}\text { Web } \\
\text { Graphs }\end{array}$ & $\begin{array}{c}\text { Google(In) } \\
\text { Berkstant(n) } \\
\text { Wikipedia2009(In) } \\
\text { WikipediatinkFr(In) } \\
\text { Hudong(In) }\end{array}$ & $\begin{array}{l}337.11 \\
42.907 \\
92.743 \\
141.99 \\
11.462\end{array}$ & $\begin{array}{l}0.01504 \\
0.02851 \\
0.00189 \\
0.02955 \\
0.00454\end{array}$ & $\begin{array}{l}14.019 \\
0.7056 \\
7.8955 \\
0.4321 \\
0.3076\end{array}$ & $\begin{array}{l}333.95 \\
\mathbf{3 4 . 3 6 0} \\
100.61 \\
144.48 \\
25.055\end{array}$ & $\begin{array}{l}0.01546 \\
0.02711 \\
0.00197 \\
0.03088 \\
0.00525\end{array}$ & $\begin{array}{l}14.129 \\
0.5673 \\
8.3468 \\
0.4451 \\
0.4596\end{array}$ & $\begin{array}{l}319.73 \\
35.906 \\
95.333 \\
136.35 \\
23.271\end{array}$ & $\begin{array}{l}0.01505 \\
0.02704 \\
0.00192 \\
0.02841 \\
0.00512\end{array}$ & $\begin{array}{l}13.993 \\
0.6067 \\
8.0388 \\
0.4202 \\
0.4378\end{array}$ & & $\begin{array}{l}0.01368 \\
0.03116 \\
0.00169 \\
0.01776 \\
0.00433\end{array}$ & $\begin{array}{l}13.845 \\
0.9478 \\
7.7498 \\
0.3174 \\
0.2508\end{array}$ \\
\hline $\begin{array}{l}\text { Biological } \\
\text { Networks }\end{array}$ & $\begin{array}{l}\text { Yeast-PPIN } \\
\text { Diseasome } \\
\text { Bio-Mouse-Gene } \\
\text { Bio-Dmela } \\
\text { Bio-WormNet-v3 }\end{array}$ & $\begin{array}{l}7.8751 \\
11.411 \\
15.990 \\
8.748 \\
12.897\end{array}$ & & $\begin{array}{l}4.0614 \\
3.3500 \\
2.4457 \\
3.3483 \\
3.7004\end{array}$ & & & $\begin{array}{l}4.1484 \\
3.3509 \\
2.4118 \\
3.3947 \\
3.7318\end{array}$ & & & & & & $\begin{array}{l}2.6321 \\
2.7445 \\
2.2557 \\
4.0968 \\
2.6066\end{array}$ \\
\hline $\begin{array}{c}\text { Product } \\
\text { co-purchasing } \\
\text { networks }\end{array}$ & $\begin{array}{l}\text { amazon0601(In) } \\
\text { amazon0505(In } \\
\text { amazon0312(In) }\end{array}$ & $\begin{array}{l}82.087 \\
104.28 \\
91.753\end{array}$ & $\begin{array}{l}0.00335 \\
0.00366 \\
0.00335\end{array}$ & $\begin{array}{l}7.1527 \\
8.5019 \\
7.5804\end{array}$ & $\begin{array}{l}81.665 \\
104.27 \\
91.416\end{array}$ & & & & & & & $\begin{array}{l}0.00374 \\
0.00412 \\
0.00430\end{array}$ & $\begin{array}{l}8.1863 \\
9.1836 \\
8.5465\end{array}$ \\
\hline $\begin{array}{l}\text { Temporal } \\
\text { Networks }\end{array}$ & $\begin{array}{l}\text { sx-mathoverflow(IIn) } \\
\text { sx-stackoverflow (In) } \\
\text { sx-superuser(In) } \\
\text { sx-askubuntu(In) }\end{array}$ & $\begin{array}{l}13.268 \\
43.321 \\
33.298 \\
46.943\end{array}$ & $\begin{array}{l}0.01537 \\
0.00341 \\
0.00409 \\
0.00669\end{array}$ & $\begin{array}{l}1.7301 \\
0.9205 \\
2.3678 \\
3.3637\end{array}$ & $\begin{array}{l}\mathbf{1 3 . 0 9 2} \\
61.452 \\
\mathbf{2 6 . 8 1 4} \\
\mathbf{3 6 . 9 3 5}\end{array}$ & $\begin{array}{l}0.01508 \\
0.00345 \\
0.00341 \\
0.00479\end{array}$ & $\begin{array}{l}\mathbf{1 . 6 8 0 9} \\
1.0709 \\
\mathbf{1 . 7 7 9 7} \\
\mathbf{2 . 3 8 7 0}\end{array}$ & $\begin{array}{l}13.769 \\
50.608 \\
31.548 \\
41.091\end{array}$ & $\begin{array}{l}0.015238 \\
0.003237 \\
0.00342 \\
0.00460\end{array}$ & & $\begin{array}{l}19.706 \\
39.654 \\
79.777 \\
106.04\end{array}$ & & $\begin{array}{l}2.4647 \\
0.8694 \\
4.5409 \\
6.2022\end{array}$ \\
\hline $\begin{array}{l}\text { Communication } \\
\text { Networks }\end{array}$ & $\begin{array}{c}\text { Email-Enron } \\
\text { Wiki-Talk(In) } \\
\text { Rec-Libimseti(In) }\end{array}$ & $\begin{array}{l}76.151 \\
325.74 \\
66.955\end{array}$ & $\begin{array}{l}0.03529 \\
0.00149 \\
0.09759\end{array}$ & $\begin{array}{l}5.2324 \\
14.119 \\
1.7695\end{array}$ & $\begin{array}{l}76.417 \\
671.976 \\
66.498\end{array}$ & $\begin{array}{l}0.03526 \\
0.00357 \\
0.09977\end{array}$ & $\begin{array}{l}5.2335 \\
25.903 \\
1.7924\end{array}$ & $\begin{array}{l}75.369 \\
654.64 \\
65.039\end{array}$ & $\begin{array}{l}0.03499 \\
0.00255 \\
0.08939\end{array}$ & $\begin{array}{l}5.1948 \\
23.816 \\
1.6652\end{array}$ & $\begin{array}{l}\mathbf{7 4 . 6 6 7} \\
670.47 \\
\mathbf{2 3 . 3 4 1}\end{array}$ & $\begin{array}{l}0.03523 \\
0.00356 \\
0.02163\end{array}$ & $\begin{array}{l}5.2075 \\
25.871 \\
0.4953\end{array}$ \\
\hline $\begin{array}{l}\text { Ground-truth } \\
\text { Networks }\end{array}$ & $\begin{array}{l}\text { Wiki-Topcats } \\
\text { com-Friendster } \\
\text { com-LiveJournal } \\
\text { com-Orkut } \\
\text { com-Youtube }\end{array}$ & $\begin{array}{l}10.906 \\
43.97 \mathrm{~K} \\
1685.8 \\
165.35 \\
83.496\end{array}$ & $\begin{array}{l}0.00190 \\
0.06351 \\
0.02289 \\
0.00548 \\
0.00177\end{array}$ & $\begin{array}{l}0.0987 \\
3427.11 \\
48.512 \\
4.7694 \\
1.1093\end{array}$ & $\begin{array}{l}10.206 \\
42.64 \mathrm{~K} \\
1738.8 \\
\mathbf{1 6 4 . 4 1} \\
82.441\end{array}$ & $\begin{array}{l}\mathbf{0 . 0 0 1 8 9} \\
0.06400 \\
0.02462 \\
0.00538 \\
0.00175\end{array}$ & $\begin{array}{l}.0951 \\
3413.5 \\
50.298 \\
4.6646 \\
1.0957\end{array}$ & $\begin{array}{l}13.254 \\
43.76 \mathrm{~K} \\
447.06 \\
315.83 \\
78.454\end{array}$ & $\begin{array}{l}0.00192 \\
0.05975 \\
0.00584 \\
0.03085 \\
0.00167\end{array}$ & $\begin{array}{l}0.1098 \\
3326.1 \\
18.469 \\
16.651 \\
1.0403\end{array}$ & & & $\begin{array}{l}0.1011 \\
411.15 \\
6.9832 \\
7.0113 \\
0.6984\end{array}$ \\
\hline $\begin{array}{c}\text { Brain } \\
\text { Networks }\end{array}$ & $\begin{array}{l}\text { Human25890-session1 } \\
\text { Human25890-session2 } \\
\text { Human2584--sesion2 } \\
\text { Human256913-session2 } 2 \\
\text { Human25886-session1 }\end{array}$ & $\begin{array}{l}17.029 \\
94.272 \\
147.06 \\
116.61 \\
113.21\end{array}$ & $\begin{array}{l}0.02172 \\
0.04548 \\
0.14826 \\
0.04524 \\
0.0525\end{array}$ & $\begin{array}{l}3.6229 \\
14.855 \\
33.184 \\
20.418 \\
22.834\end{array}$ & $\begin{array}{l}17.843 \\
96.959 \\
108.04 \\
119.75 \\
116.77\end{array}$ & $\begin{array}{l}0.02264 \\
0.04769 \\
0.05325 \\
0.04718 \\
0.05476\end{array}$ & $\begin{array}{l}3.7572 \\
15.296 \\
20.661 \\
20.982 \\
23.393\end{array}$ & $\begin{array}{l}\mathbf{1 5 . 1 5 0} \\
61.328 \\
147.63 \\
\mathbf{8 5 . 3 2 6} \\
\mathbf{6 4 . 9 5 1}\end{array}$ & $\begin{array}{l}\mathbf{0 . 0 2 0 6 8} \\
0.01488 \\
0.14423 \\
0.04042 \\
0.03826\end{array}$ & & & & $\begin{array}{l}3.7439 \\
5.8781 \\
9.8670 \\
11.574 \\
12.051\end{array}$ \\
\hline
\end{tabular}

and it gives the 'best' fit for 22 out of 50 data sets considered in this study.

310

Other than the statistical comparison, visual inspections of the fits of different models are used as an essential tool for model validation and comparison. This motivates us to draw the plots of the original frequency distribution and the estimated frequencies (in log-log scale) by GLM family of distributions and the frequency estimated by other competing distributions used in this paper viz. Lomax, log-normal, power-law, power-law cutoff, and Exponential distributions over all the networks under consideration. Figures 6 and 7 provide the plots of different models in the log-log scale for twelve network data sets from other domains. It is visually clear from the Figures 6 and 7 that the proposed GLM models provide a better fit in comparison to the other single heavy-tailed and life distributions under considerations for most of the network data sets. Pictorial representation from the log-log plots shows that the proposed model always passes through the middle of the scatter plot of the observed distribution compared to the competing heavy-tailed distributions in very few cases. From the practitioner's point of view, the proposed fitted distribution can easily capture the spatial structure of the network by simulating the model parameters. The new concept of the GLM family of distributions provides a better and efficient fit for the entire degree distribution of real-world heavy-tailed complex networks than the other competitive heavy-tailed distributions.

\section{Discussion and Conclusion}

Learning from complex networks (such as social, information, biological, temporal, and brain networks) is a difficult proposition. Many studies on complex networks focus on network topology and network statistical mechanics from a scale-free perspective. 
Table 6. Performance evaluation of commonly used heavy-tailed models over 50 real-world network data

\begin{tabular}{|c|c|c|c|c|c|c|c|c|c|c|c|c|c|c|c|c|}
\hline \multicolumn{2}{|r|}{$\begin{array}{l}\text { Data } \\
\text { sets }\end{array}$} & \multicolumn{3}{|c|}{ Lomax } & \multicolumn{3}{|c|}{ power-law } & \multicolumn{3}{|c|}{ Log-normal } & \multicolumn{3}{|c|}{$\begin{array}{c}\text { power-law } \\
\text { cutoff }\end{array}$} & \multicolumn{3}{|c|}{ Exponential } \\
\hline & & RMSE & KLD & MAE & RMSE & KLD & MAE & RMSE & KLD & MAE & RMSE & KLD & MAE & RMSE & KLD & MAE \\
\hline $\begin{array}{l}\text { Social } \\
\text { Networks }\end{array}$ & 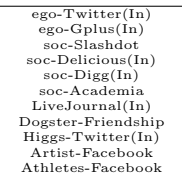 & $\begin{array}{l}29.366 \\
10.491 \\
32.065 \\
91.993 \\
24.841 \\
48.951 \\
1764.9 \\
36.449 \\
20.609 \\
12.923 \\
9.2379\end{array}$ & $\begin{array}{l}0.01354 \\
0.06444 \\
0.014102 \\
0.01326 \\
0.02391 \\
0.01019 \\
0.02111 \\
0.01700 \\
0.00793 \\
0.01199 \\
0.00966\end{array}$ & $\begin{array}{l}2.4701 \\
0.3033 \\
2.468 \\
4.8060 \\
1.06269 \\
1.6178 \\
54.400 \\
1.0755 \\
0.4621 \\
2.6673 \\
1.8260\end{array}$ & $\begin{array}{l}204.35 \\
53.064 \\
247.87 \\
349.66 \\
208.01 \\
229.54 \\
5025.2 \\
358.27 \\
260.32 \\
100.49 \\
100.16\end{array}$ & $\begin{array}{l}0.1831 \\
0.2299 \\
0.1007 \\
0.2021 \\
0.1601 \\
0.2027 \\
0.1614 \\
0.2926 \\
0.2492 \\
0.1643 \\
0.2049\end{array}$ & $\begin{array}{l}10.847 \\
0.921 \\
10.074 \\
14.867 \\
4.2185 \\
6.3889 \\
127.98 \\
5.6815 \\
4.8938 \\
14.552 \\
13.387\end{array}$ & $\begin{array}{l}53.863 \\
10.155 \\
237.63 \\
281.34 \\
69.438 \\
91.003 \\
3473.6 \\
42.539 \\
41.955 \\
24.071 \\
15.461\end{array}$ & $\begin{array}{l}0.0169 \\
0.0678 \\
0.1058 \\
0.0579 \\
0.0552 \\
0.0169 \\
0.00355 \\
0.0272 \\
0.0134 \\
0.0154 \\
0.0127\end{array}$ & $\begin{array}{l}2.9494 \\
0.2523 \\
10.549 \\
10.781 \\
1.9087 \\
2.0921 \\
70.64 \\
1.1599 \\
0.5595 \\
3.026 \\
2.5674\end{array}$ & $\begin{array}{l}68.004 \\
30.025 \\
19.598 \\
66.896 \\
65.73 \\
62.376 \\
808.79 \\
182.47 \\
118.23 \\
56.281 \\
25.099\end{array}$ & $\begin{array}{l}0.0397 \\
0.1475 \\
0.0075 \\
0.0185 \\
0.0441 \\
0.0255 \\
0.0101 \\
0.1862 \\
0.0914 \\
0.0458 \\
0.0324\end{array}$ & $\begin{array}{l}4.1974 \\
0.6821 \\
1.3599 \\
4.2366 \\
1.99042 \\
1.9845 \\
24.501 \\
4.1579 \\
2.8051 \\
6.6452 \\
4.3180\end{array}$ & $\begin{array}{l}157.98 \\
50.098 \\
434.25 \\
535.11 \\
204.50 \\
198.11 \\
7017.9 \\
165.19 \\
134.68 \\
88.651 \\
28.388\end{array}$ & $\begin{array}{l}0.2733 \\
1.3131 \\
0.6381 \\
0.4626 \\
0.8907 \\
0.9924 \\
0.3449 \\
0.4765 \\
0.3163 \\
0.169 \\
0.1958\end{array}$ & $\begin{array}{l}11.567 \\
1.828 \\
22.275 \\
25.304 \\
8.0015 \\
6.6739 \\
186.01 \\
5.4623 \\
4.4689 \\
13.623 \\
6.2610\end{array}$ \\
\hline $\begin{array}{l}\text { Citation } \\
\text { Networks }\end{array}$ & $\begin{array}{l}\text { cit-HepTh(In) } \\
\text { cit-HepPh(In) } \\
\text { cit-Patents(In) } \\
\text { cit-Citeseer(In) }\end{array}$ & $\begin{array}{l}7.9393 \\
21.303 \\
2577.5 \\
28.032\end{array}$ & $\begin{array}{l}0.01585 \\
0.01317 \\
0.00192 \\
0.00278\end{array}$ & $\begin{array}{l}0.7585 \\
3.1135 \\
230.35 \\
3.3902\end{array}$ & $\begin{array}{l}73.531 \\
128.55 \\
27.5 \mathrm{~K} \\
889.88\end{array}$ & $\begin{array}{l}0.1741 \\
0.1825 \\
0.2266 \\
0.3308\end{array}$ & $\begin{array}{l}4.0821 \\
13.234 \\
2049.5 \\
49.467\end{array}$ & $\begin{array}{l}22.59 \\
44.951 \\
9612.7 \\
353.26\end{array}$ & $\begin{array}{l}0.0255 \\
0.0189 \\
0.0192 \\
0.0299\end{array}$ & $\begin{array}{l}2.331 \\
4.405 \\
725.71 \\
21.921\end{array}$ & $\begin{array}{l}25.42 \\
36.887 \\
2424.5 \\
195.67\end{array}$ & $\begin{array}{l}0.0464 \\
0.0221 \\
0.0061 \\
0.0131\end{array}$ & $\begin{array}{l}2.816 \\
4.5287 \\
271.89 \\
13.877\end{array}$ & $\begin{array}{l}58.74 \\
107.32 \\
13.2 \mathrm{~K} \\
629.02\end{array}$ & $\begin{array}{l}0.2778 \\
0.1801 \\
0.0659 \\
0.1486\end{array}$ & $\begin{array}{l}.5286 \\
14.145 \\
1147.5 \\
44.301\end{array}$ \\
\hline $\begin{array}{l}\text { Collaboration } \\
\text { Networks }\end{array}$ & $\begin{array}{l}\text { ca-CondMat } \\
\text { ca-AstroPh } \\
\text { ca-GrQc } \\
\text { ca-HepPh } \\
\text { ca-HepTh }\end{array}$ & $\begin{array}{l}36.094 \\
32.799 \\
35.935 \\
19.607 \\
61.797\end{array}$ & $\begin{array}{l}0.00814 \\
0.03457 \\
0.04013 \\
0.07266 \\
0.01353\end{array}$ & $\begin{array}{l}7.3904 \\
7.4448 \\
12.286 \\
4.7763 \\
20.391\end{array}$ & $\begin{array}{l}107.86 \\
92.255 \\
124.24 \\
75.071 \\
268.91\end{array}$ & $\begin{array}{l}0.1025 \\
0.1753 \\
0.2554 \\
0.1769 \\
0.2346\end{array}$ & $\begin{array}{l}26.092 \\
15.158 \\
27.137 \\
8.0906 \\
66.108\end{array}$ & $\begin{array}{l}42.665 \\
28.209 \\
30.184 \\
29.993 \\
55.618\end{array}$ & $\begin{array}{l}0.0082 \\
0.0312 \\
0.0515 \\
0.1011 \\
0.0178\end{array}$ & $\begin{array}{l}6.5746 \\
6.8565 \\
12.148 \\
6.8958 \\
21.032\end{array}$ & $\begin{array}{l}62.929 \\
50.604 \\
58.305 \\
50.717 \\
89.425\end{array}$ & $\begin{array}{l}0.0287 \\
0.0384 \\
0.0659 \\
0.1128 \\
0.0245\end{array}$ & $\begin{array}{l}13.362 \\
7.459 \\
18.259 \\
8.2477 \\
27.613\end{array}$ & $\begin{array}{l}64.985 \\
68.185 \\
69.169 \\
89.936 \\
109.96\end{array}$ & $\begin{array}{l}0.0472 \\
0.1235 \\
0.1418 \\
0.5187 \\
0.0551\end{array}$ & $\begin{array}{l}16.873 \\
14.361 \\
25.759 \\
17.589 \\
43.882\end{array}$ \\
\hline $\begin{array}{l}\text { Web } \\
\text { Graphs }\end{array}$ & $\begin{array}{c}\text { Google(In) } \\
\text { Berkstann(In) } \\
\text { Wikipedia2009(In) } \\
\text { WikipediaLinkF(IIn) } \\
\text { Hudong(In) }\end{array}$ & $\begin{array}{l}337.68 \\
105.20 \\
103.94 \\
146.14 \\
25.163\end{array}$ & $\begin{array}{l}0.01546 \\
0.0346 \\
0.00197 \\
0.03082 \\
0.00525\end{array}$ & $\begin{array}{l}14.201 \\
1.1962 \\
8.5289 \\
0.4465 \\
0.4600\end{array}$ & $\begin{array}{l}1809.1 \\
615.03 \\
4371.9 \\
248.09 \\
587.21\end{array}$ & $\begin{array}{l}0.124 \\
0.1863 \\
0.1352 \\
0.1518 \\
0.0868\end{array}$ & $\begin{array}{l}45.023 \\
4.0722 \\
164.58 \\
0.7857 \\
4.6828\end{array}$ & $\begin{array}{l}1514.5 \\
185.04 \\
2720.9 \\
240.18 \\
746.47\end{array}$ & $\begin{array}{l}0.0878 \\
0.1002 \\
0.0798 \\
0.0543 \\
0.1593\end{array}$ & $\begin{array}{l}40.067 \\
2.0198 \\
116.43 \\
0.5234 \\
6.5493\end{array}$ & $\begin{array}{l}188.01 \\
322.63 \\
781.87 \\
121.31 \\
75.362\end{array}$ & $\begin{array}{l}0.0157 \\
0.1037 \\
0.0082 \\
0.0022 \\
0.0063\end{array}$ & $\begin{array}{l}9.6549 \\
2.8203 \\
35.531 \\
0.5006 \\
0.8323\end{array}$ & $\begin{array}{l}2589.4 \\
595.53 \\
4727.1 \\
534.61 \\
1494.8\end{array}$ & $\begin{array}{l}0.4419 \\
0.738 \\
0.3431 \\
1.0217 \\
1.1798\end{array}$ & $\begin{array}{l}76.441 \\
6.6185 \\
213.66 \\
2.0471 \\
15.836\end{array}$ \\
\hline $\begin{array}{l}\text { Biological } \\
\text { Networks }\end{array}$ & $\begin{array}{c}\text { Yeast-PPIN } \\
\text { Diseasome } \\
\text { Bio-Mouse-Gene } \\
\text { Bio-Dmela } \\
\text { Bio-WormNet-v3 }\end{array}$ & $\begin{array}{l}12.651 \\
12.451 \\
14.654 \\
10.579 \\
13.018\end{array}$ & $\begin{array}{l}0.02389 \\
0.10202 \\
0.19473 \\
0.01759 \\
0.09249\end{array}$ & $\begin{array}{l}5.4529 \\
3.455 \\
2.3919 \\
3.8219 \\
3.7468\end{array}$ & $\begin{array}{l}75.325 \\
26.006 \\
41.371 \\
143.71 \\
46.259\end{array}$ & $\begin{array}{l}0.1999 \\
0.2248 \\
0.4566 \\
0.1907 \\
0.2761\end{array}$ & $\begin{array}{l}19.013 \\
5.3567 \\
3.9018 \\
21.415 \\
6.8867\end{array}$ & $\begin{array}{l}29.928 \\
23.282 \\
17.199 \\
46.271 \\
17.726\end{array}$ & $\begin{array}{l} \\
0.0496 \\
0.1552 \\
0.1878 \\
0.0426 \\
0.0851\end{array}$ & $\begin{array}{l}9.3869 \\
4.8906 \\
2.5372 \\
9.2857 \\
3.9352\end{array}$ & $\begin{array}{l}4.9595 \\
9.3332 \\
9.277 \\
24.091 \\
6.7764\end{array}$ & $\begin{array}{l}0.0175 \\
0.0822 \\
\mathbf{0 . 0 9 4 3} \\
0.0162 \\
\mathbf{0 . 0 4 1 9}\end{array}$ & $\begin{array}{l}2.9178 \\
2.8587 \\
1.6036 \\
5.0541 \\
2.2424\end{array}$ & $\begin{array}{l}45.462 \\
31.709 \\
31.649 \\
86.659 \\
40.795\end{array}$ & $\begin{array}{l}0.1234 \\
0.2979 \\
0.4882 \\
0.1724 \\
0.3082\end{array}$ & $\begin{array}{l}13.786 \\
5.7013 \\
3.6376 \\
18.048 \\
7.0826\end{array}$ \\
\hline $\begin{array}{c}\text { Product } \\
\text { co-purchasing } \\
\text { networks }\end{array}$ & $\begin{array}{l}\text { amazon0601(In) } \\
\text { amazon0505(In) } \\
\text { amazon0312(In) }\end{array}$ & $\begin{array}{l}147.602 \\
114.048 \\
92.525\end{array}$ & $\begin{array}{l}0.00695 \\
0.00499 \\
0.00495\end{array}$ & $\begin{array}{l}10.928 \\
8.7882 \\
8.5742\end{array}$ & $\begin{array}{l}1495.4 \\
1572.9 \\
1564.4\end{array}$ & $\begin{array}{l}0.2708 \\
0.2463 \\
0.2425\end{array}$ & $\begin{array}{l}70.281 \\
73.003 \\
71.875\end{array}$ & $\begin{array}{l}286.61 \\
358.59 \\
338.03\end{array}$ & $\begin{array}{l}0.0102 \\
0.0125 \\
0.0116\end{array}$ & $\begin{array}{l}16.881 \\
19.123 \\
17.742\end{array}$ & $\begin{array}{l}297.39 \\
260.85 \\
273.39\end{array}$ & $\begin{array}{l}0.0382 \\
0.0342 \\
0.0352\end{array}$ & $\begin{array}{l}22.199 \\
20.136 \\
20.381\end{array}$ & $\begin{array}{l}308.32 \\
390.13 \\
383.82\end{array}$ & $\begin{array}{l}0.0574 \\
0.0628 \\
0.0639\end{array}$ & $\begin{array}{l}24.114 \\
26.178 \\
26.299\end{array}$ \\
\hline $\begin{array}{l}\text { Temporal } \\
\text { Networks }\end{array}$ & $\begin{array}{l}\text { sx-mathoverflow (In) } \\
\text { sx-stackoverflow (In) } \\
\text { sx-superuser(In) } \\
\text { sx-askubutuntu(In) }\end{array}$ & $\begin{array}{l}38.764 \\
62.254 \\
136.85 \\
176.58\end{array}$ & $\begin{array}{l}0.02621 \\
0.00345 \\
0.01045 \\
0.01707\end{array}$ & $\begin{array}{l}3.7877 \\
1.0741 \\
6.8313 \\
9.3509\end{array}$ & $\begin{array}{l}213.91 \\
1877.5 \\
900.04 \\
949.66\end{array}$ & $\begin{array}{l}0.2131 \\
0.2016 \\
0.1808 \\
0.2091\end{array}$ & $\begin{array}{l}13.600 \\
14.007 \\
33.837 \\
39.419\end{array}$ & $\begin{array}{l}41.934 \\
341.96 \\
243.42 \\
212.91\end{array}$ & $\begin{array}{l}0.0634 \\
0.0286 \\
0.0616 \\
0.0649\end{array}$ & $\begin{array}{l}5.3161 \\
4.4267 \\
13.199 \\
12.451\end{array}$ & $\begin{array}{l}92.603 \\
740.18 \\
354.72 \\
389.14\end{array}$ & $\begin{array}{l}0.0861 \\
0.0685 \\
0.0570 \\
0.0719\end{array}$ & $\begin{array}{l}7.9912 \\
7.2275 \\
16.613 \\
20.113\end{array}$ & $\begin{array}{l}129.69 \\
1324.4 \\
609.40 \\
555.44\end{array}$ & $\begin{array}{l}0.4636 \\
0.6829 \\
0.3891 \\
0.3433\end{array}$ & $\begin{array}{l}15.172 \\
19.362 \\
34.655 \\
33.693\end{array}$ \\
\hline $\begin{array}{l}\text { Communication } \\
\text { Networks }\end{array}$ & $\begin{array}{c}\text { Email-Enron } \\
\text { Wiki-Talk(In) } \\
\text { Rec-Libimseti(In) }\end{array}$ & $\begin{array}{l}76.155 \\
671.76 \\
66.434\end{array}$ & $\begin{array}{l}0.03531 \\
0.00357 \\
0.09978\end{array}$ & $\begin{array}{l}5.2347 \\
25.898 \\
1.7923\end{array}$ & $\begin{array}{l}246.51 \\
9669.4 \\
77.081\end{array}$ & $\begin{array}{l}0.1779 \\
0.3376 \\
0.2198\end{array}$ & $\begin{array}{l}14.886 \\
293.63 \\
2.1486\end{array}$ & $\begin{array}{l}121.47 \\
7978.6 \\
87.472\end{array}$ & $\begin{array}{l}0.0873 \\
0.1902 \\
0.0755\end{array}$ & $\begin{array}{l}8.445 \\
246.26 \\
1.4021\end{array}$ & $\begin{array}{l}95.468 \\
672.32 \\
28.059\end{array}$ & $\begin{array}{l}0.0689 \\
0.0036 \\
0.0359\end{array}$ & $\begin{array}{l}7.664 \\
25.905 \\
0.6971\end{array}$ & $\begin{array}{l}230.41 \\
16.5 \mathrm{~K} \\
166.18\end{array}$ & $\begin{array}{l}0.5405 \\
0.4879 \\
0.8547\end{array}$ & $\begin{array}{l}18.139 \\
542.31 \\
3.9402\end{array}$ \\
\hline $\begin{array}{l}\text { Ground-truth } \\
\text { Networks }\end{array}$ & $\begin{array}{l}\text { Wiki-Topcats } \\
\text { com-Friendster } \\
\text { com-LiveJournal } \\
\text { com-Orkut } \\
\text { com-Youtube }\end{array}$ & $\begin{array}{l}14.955 \\
41.69 \mathrm{~K} \\
1741.3 \\
207.43 \\
81.409\end{array}$ & $\begin{array}{l}0.00201 \\
0.06401 \\
0.02462 \\
0.01049 \\
0.00175\end{array}$ & $\begin{array}{l}0.1347 \\
3385.2 \\
50.318 \\
9.9761 \\
1.0862\end{array}$ & $\begin{array}{l}565.21 \\
71.5 \mathrm{~K} \\
4102.9 \\
2443.6 \\
1380.5\end{array}$ & $\begin{array}{l}0.1377 \\
0.1498 \\
0.1823 \\
0.5498 \\
0.1342\end{array}$ & $\begin{array}{l}2.6145 \\
4575.6 \\
106.85 \\
80.712 \\
15.690\end{array}$ & $\begin{array}{l}272.99 \\
101 \mathrm{~K} \\
2629.9 \\
452.92 \\
1422.2\end{array}$ & $\begin{array}{l}0.0464 \\
0.0762 \\
0.0299 \\
0.0259 \\
0.1416\end{array}$ & $\begin{array}{l}1.5159 \\
4022.8 \\
51.656 \\
19.624 \\
17.219\end{array}$ & $\begin{array}{l}389.86 \\
17.8 \mathrm{~K} \\
497.89 \\
261.75 \\
143.79\end{array}$ & $\begin{array}{l}0.0629 \\
0.0052 \\
0.0104 \\
0.0479 \\
0.0045\end{array}$ & $\begin{array}{l}2.2289 \\
1025.5 \\
18.559 \\
16.197 \\
2.2564\end{array}$ & $\begin{array}{l}832.23 \\
193 K \\
5230.3 \\
228.83 \\
2515.4\end{array}$ & $\begin{array}{l}0.6767 \\
0.7216 \\
0.2889 \\
0.0099 \\
0.6241\end{array}$ & $\begin{array}{l}5.8936 \\
10.1 \mathrm{~K} \\
139.74 \\
14.496 \\
31.101\end{array}$ \\
\hline $\begin{array}{c}\text { Brain } \\
\text { Networks }\end{array}$ & $\begin{array}{l}\text { Human25890-session1 } \\
\text { Human2589-session2 } \\
\text { Human25864-ession2 } \\
\text { Human25913-session2 } \\
\text { Human2588-ession } 2586 \text { ession1 }\end{array}$ & $\begin{array}{l}19.545 \\
97.122 \\
111.45 \\
119.54 \\
116.78\end{array}$ & $\begin{array}{l}0.02264 \\
0.04774 \\
0.05335 \\
0.04719 \\
0.05476\end{array}$ & $\begin{array}{l}4.0023 \\
15.303 \\
20.876 \\
20.971 \\
23.396\end{array}$ & $\begin{array}{l}305.41 \\
794.95 \\
1120.3 \\
904.76 \\
978.66\end{array}$ & $\begin{array}{l}0.3397 \\
0.4462 \\
0.4967 \\
0.2764 \\
0.4664\end{array}$ & $\begin{array}{l}22.537 \\
50.379 \\
68.172 \\
58.999 \\
69.517\end{array}$ & $\begin{array}{l}27.703 \\
78.483 \\
83.489 \\
99.615 \\
89.805\end{array}$ & $\begin{array}{l}0.0222 \\
0.0471 \\
0.0495 \\
0.0292 \\
0.0568\end{array}$ & $\begin{array}{l}4.1822 \\
14.289 \\
18.215 \\
15.796 \\
20.819\end{array}$ & $\begin{array}{l}65.972 \\
83.707 \\
106.20 \\
223.39 \\
102.34\end{array}$ & $\begin{array}{l}0.0509 \\
0.0162 \\
0.0156 \\
0.0219 \\
0.0154\end{array}$ & $\begin{array}{l}7.8146 \\
6.709 \\
9.7828 \\
15.122 \\
11.296\end{array}$ & $\begin{array}{l}92.649 \\
183.06 \\
212.96 \\
440.58 \\
207.52\end{array}$ & $\begin{array}{l}0.2272 \\
0.1701 \\
0.1605 \\
0.331 \\
0.1354\end{array}$ & $\begin{array}{l}15.977 \\
26.302 \\
33.272 \\
59.629 \\
33.287\end{array}$ \\
\hline
\end{tabular}
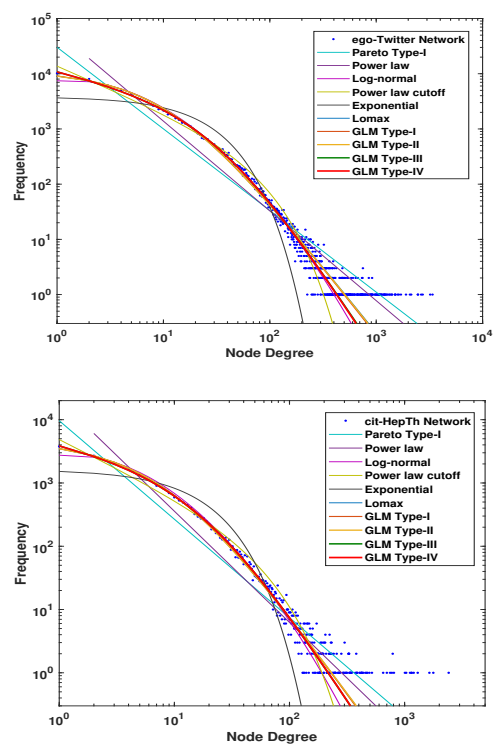

(a)
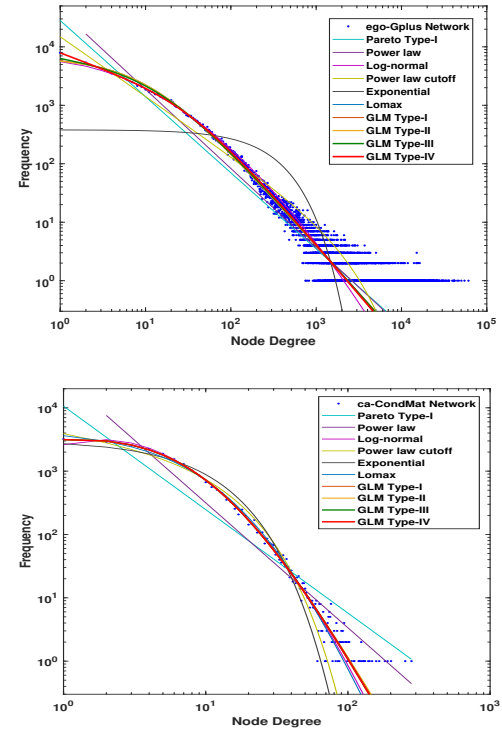

(b)

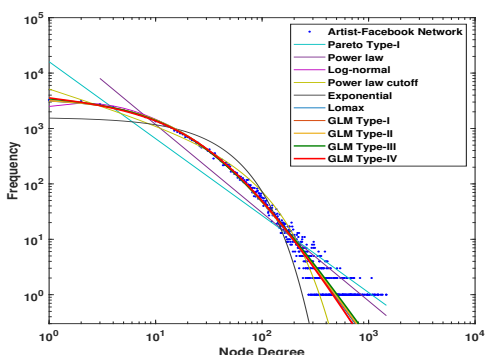

(c)

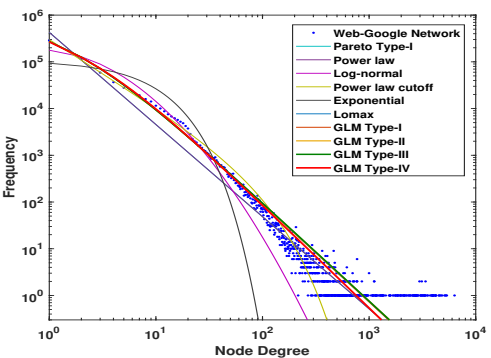

Fig 6. Plots of degree distributions of (a) ego-Twitter(In), (b) ego-Gplus(In), (c)

Artist-Facebook, (d) cit-HepTh(In), (e) ca-CondMat and (f) Google(In) in log-log scale. 

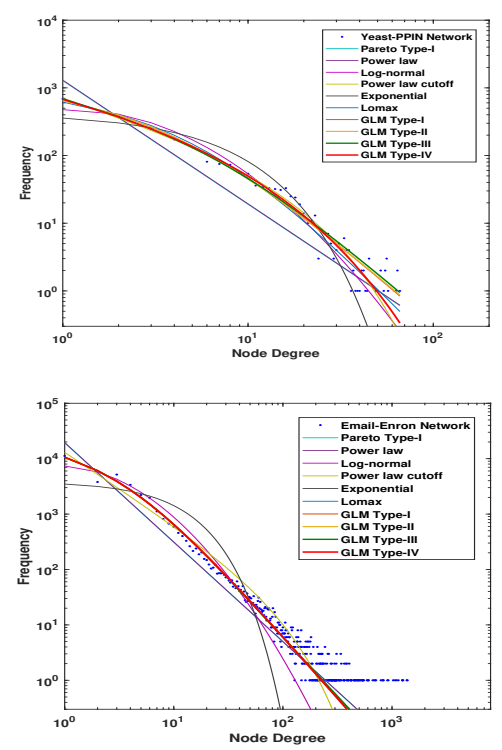

(a)
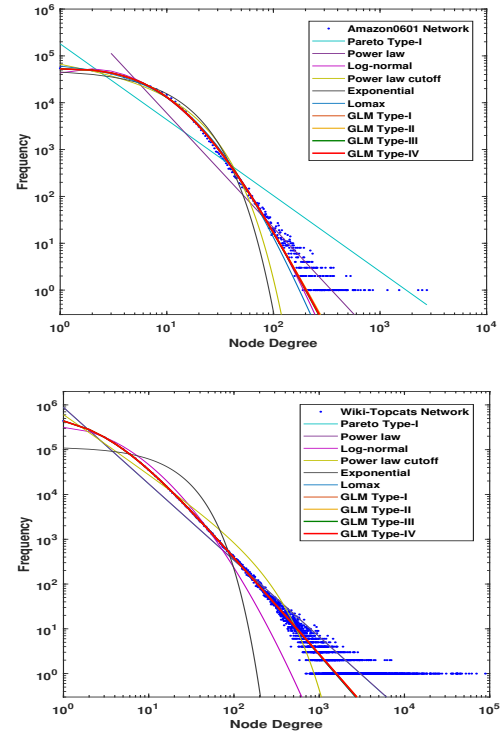

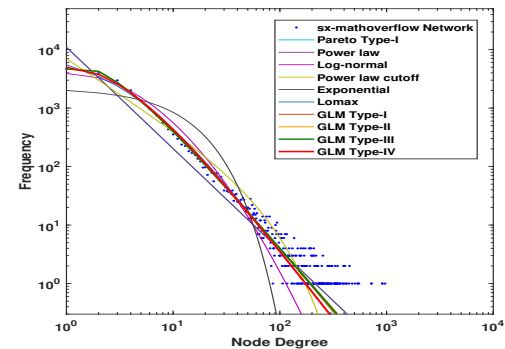

(c)

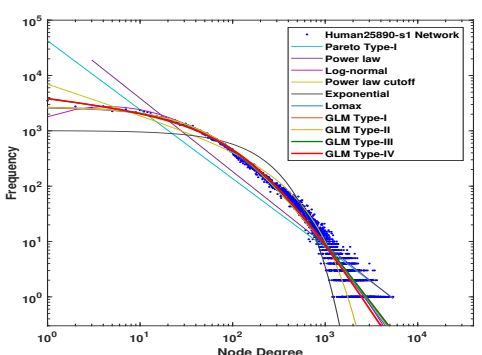

Fig 7. Plots of degree distributions of (a) Yeast-PPIN, (b) amazon0601(In), (c) sx-mathoverflow(In), (d) Email-Enron, (e) Wiki-Topcats and (f) Human25890-session1 in $\log -\log$ scale.

However, the putative scale-free nature of real-world networks has generated a lot of interest in the past two decades. Therefore, most of the recent studies emphasized on the testing of the degree distribution of networks for power-law tails 18, 22, 38 40]. As the question "Are real-world networks scale-free" has important philosophical and conceptual consequences, we have considered this question methodologically. This paper explores a journey towards non-scale-freeness and proposes a new family of generalized Lomax models with nonlinear exponents in the shape parameter for efficient modeling of the heavy-tailed behavior of complex networks. The proposed GLM models provide a better fitting to the whole degree distributions of real-world complex networks than other well-known heavy-tailed distributions which could be thought of as a constructive answer to the overarching question raised in the literature: "Are complex networks scale-free? If not then what?" 18,22,40]. With this current paper, we hope to have contributed to this recent methodological progress.

The generalization of Lomax model provides us greater insight into probability distribution theory as well. Interestingly, several well-known probability distributions such as Lomax, Exponential, Rayleigh, Weibull, Gompertz and Benini and GLM Type-IV (MLM Distribution proposed in [16, 17]) arise as particular cases of GLM family of distributions whereas all other GLM type models are completely new. Another important insight from distribution theory point of view is that one may come up with several other choices of $g(z)$ and build new probability distributions from the GLM family of distributions. Theoretical analysis suggests that these newly introduced GLM models belong to the maximum domain of attraction of the Frechet distribution. It has also been observed that the proposed family of distributions (GLM) is right-tail equivalent to the Pareto distribution. We theoretically show that these models are heavy-tailed and long-tailed distributions. These models are highly efficient in modeling the whole degree distribution of real-world complex networks, unlike popularly used power-law and other probabilistic models. From the practitioner's point of view, the proposed GLM distributions enable us to characterize the patterns of the degree distributions accurately, leading to the effective modeling of the large-scale networks. 
The proposed GLM models satisfy several extreme value properties and desired

inferential characteristics. The closeness between this heavy-tailed GLM family with life distributions is also discussed. Thus, the current paper may fulfill the search for a new probability distribution for modelling heavy-tailed real-world networks which are rarely scale-free as discussed in [11. The proposed idea of the 'shape parameter-based' generalization for the Lomax distribution presented in this paper can also be applied to generalize other similar income and size distributions, namely Dagum, Burr, Beta-prime, and Log-logistic distributions. It is important to note from the experimental results and evaluations that four GLM Type models mostly outperforms all the well-known degree distribution models for a wide variety of real-world network data sets. Though the choice of $g(z)$ is still very subjective and based on the data set type; one may choose the desired Type from the GLM family of distributions. An immediate extension of this work is to verify these newly introduced GLM family of distributions for modeling other centrality measures (betweenness, closeness, etc.) of heavy-tailed real-world networks.

\section{Data and Codes}

For the sake of reproducibility, all codes and data sets are made publicly available at https://github.com/tanujit123/nonscalefree.

\section{References}

1. Albert R, Barabási AL. Statistical mechanics of complex networks. Reviews of modern physics. 2002;74(1):47.

2. Maji G, Dutta A, Malta MC, Sen S. Identifying and ranking super spreaders in real world complex networks without influence overlap. Expert Systems with Applications. 2021; p. 115061.

3. Deppman A, Andrade-Ii EO. Emergency of Tsallis statistics in fractal networks. PloS one. 2021;16(9):e0257855.

4. Adamic LA, Huberman BA. Power-law distribution of the world wide web. Science. 2000;287(5461):2115-2115.

5. Albert R, Jeong H, Barabási AL. Error and attack tolerance of complex networks. Nature. 2000;406(6794):378-382.

6. Zhang L, Small M, Judd K. Exactly scale-free scale-free networks. Physica A: Statistical Mechanics and its Applications. 2015;433:182-197.

7. Amaral LAN, Scala A, Barthelemy M, Stanley HE. Classes of small-world networks. Proceedings of the national academy of sciences. 2000;97(21):11149-11152.

8. Barabasi AL. The origin of bursts and heavy tails in human dynamics. Nature. 2005;435(7039):207-211.

9. Barabási AL, Albert R. Emergence of scaling in random networks. Science. 1999;286(5439):509-512.

10. Newman ME. The structure of scientific collaboration networks. Proceedings of the national academy of sciences. 2001;98(2):404-409. 
11. Clauset A, Shalizi CR, Newman ME. Power-law distributions in empirical data. SIAM review. 2009;51(4):661-703.

12. Lykousas N, Patsakis C. Large-scale analysis of grooming in modern social networks. Expert Systems with Applications. 2021;176:114808.

13. Hartmann B, Sugár V. Searching for small-world and scale-free behaviour in long-term historical data of a real-world power grid. Scientific Reports. 2021;11(1):1-10.

14. Chattopadhyay S, Murthy C, Pal SK. Fitting truncated geometric distributions in large scale real world networks. Theoretical Computer Science. 2014;551:22-38.

15. Voitalov I, van der Hoorn P, van der Hofstad R, Krioukov D. Scale-free networks well done. Physical Review Research. 2019;1(3):033034.

16. Chattopadhyay S, Chakraborty T, Ghosh K, Das AK. Uncovering patterns in heavy-tailed networks: A journey beyond scale-free. In: 8th ACM IKDD CODS and 26th COMAD. ACM; 2021. p. 136-144.

17. Chattopadhyay S, Chakraborty T, Ghosh K, Das AK. Modified Lomax Model: A heavy-tailed distribution for fitting large-scale real-world complex networks. Social Network Analysis and Mining. 2021;11(1):1-24.

18. Broido AD, Clauset A. Scale-free networks are rare. Nature communications. 2019;10(1):1-10.

19. Stumpf MP, Wiuf C, May RM. Subnets of scale-free networks are not scale-free: sampling properties of networks. Proceedings of the National Academy of Sciences. 2005;102(12):4221-4224.

20. Seshadri M, Machiraju S, Sridharan A, Bolot J, Faloutsos C, Leskove J. Mobile call graphs: beyond power-law and lognormal distributions. In: Proceedings of the 14th ACM SIGKDD international conference on Knowledge discovery and data mining; 2008. p. 596-604.

21. Sala A, Zheng H, Zhao BY, Gaito S, Rossi GP. Brief announcement: revisiting the power-law degree distribution for social graph analysis. In: Proceedings of the 29th ACM SIGACT-SIGOPS symposium on Principles of distributed computing; 2010. p. 400-401.

22. Holme P. Rare and everywhere: Perspectives on scale-free networks. Nature communications. 2019;10(1):1-3.

23. Stumpf MP, Porter MA. Critical truths about power laws. Science. 2012;335(6069):665-666.

24. Kaviani S, Sohn I. Application of complex systems topologies in artificial neural networks optimization: An overview. Expert Systems with Applications. 2021; p. 115073.

25. Newman ME. The structure and function of complex networks. SIAM review. 2003;45(2):167-256.

26. Newman ME. Power laws, Pareto distributions and Zipf's law. Contemporary physics. 2005;46(5):323-351.

27. Lomax K. Business failures: Another example of the analysis of failure data. Journal of the American Statistical Association. 1954;49(268):847-852. 
28. Hassan AS, Al-Ghamdi AS. Optimum step stress accelerated life testing for Lomax distribution. Journal of Applied Sciences Research. 2009;5(12):2153-2164.

29. Cramer E, Schmiedt AB. Progressively Type-II censored competing risks data from Lomax distributions. Computational Statistics \& Data Analysis. 2011;55(3):1285-1303.

30. Fares ASM, Gopal VVH. The generalized double Lomax distribution with applications. Statistica. 2016;76(4):341-352.

31. Balkema AA, De Haan L. Residual life time at great age. The Annals of probability. 1974; p. $792-804$.

32. Ahsanullah M. Record values of the Lomax distribution. Statistica Neerlandica. 1991;45(1):21-29.

33. Al-Awadhi S, Ghitany M. Statistical properties of Poisson-Lomax distribution and its application to repeated accidents data. Journal of Applied Statistical Science. 2001;10(4):365-372.

34. Nayak TK. Multivariate Lomax distribution: properties and usefulness in reliability theory. Journal of Applied Probability. 1987;24(1):170-177.

35. Hosking JR, Wallis JR. Parameter and quantile estimation for the generalized Pareto distribution. Technometrics. 1987;29(3):339-349.

36. Gradshteyn I, Ryzhik I. Handbook of mathematical functions; 1965.

37. Embrechts P, Klüppelberg C, Mikosch T. Modelling extremal events: for insurance and finance. vol. 33. Springer Science \& Business Media; 2013.

38. Serafino M, Cimini G, Maritan A, Rinaldo A, Suweis S, Banavar JR, Caldarelli G. True scale-free networks hidden by finite size effects. Proceedings of the National Academy of Sciences. 2021;118(2).

39. Zhou B, Meng X, Stanley HE. Power-law distribution of degree-degree distance: A better representation of the scale-free property of complex networks. Proceedings of the National Academy of Sciences. 2020;117(26):14812-14818.

40. Artico I, Smolyarenko I, Vinciotti V, Wit EC. How rare are power-law networks really? Proceedings of the Royal Society A. 2020;476(2241):20190742. 